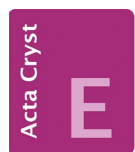

CRYSTALLOGRAPHIC COMMUNICATIONS

ISSN 2056-9890

Received 13 December 2021

Accepted 4 January 2022

Edited by J. T. Mague, Tulane University, USA

₹ Authors contributed equally to this work.

Keywords: crystal structure; quadruple bond; molybdenum; delta bond.

CCDC reference: 2132473

Supporting information: this article has supporting information at journals.iucr.org/e

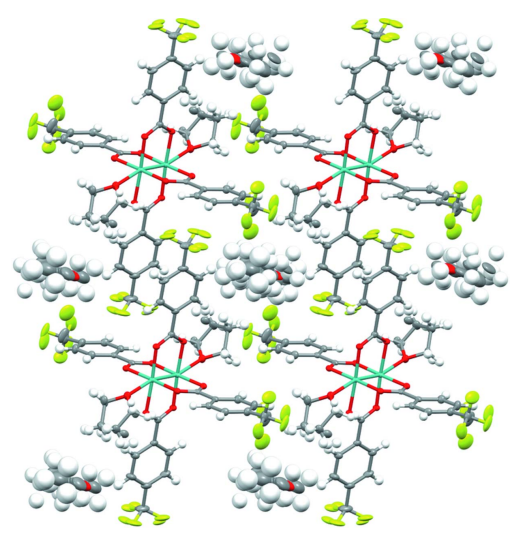

\section{Crystal structure of a trifluoromethyl benzoato quadruple-bonded dimolybdenum complex}

\author{
Elisabeth Aigeldinger,‡ Lilliana Brandao,‡ Troy Powell,‡ Alaina C. Hartnett, Rui \\ Sun, Dilek K. Dogutan* and Shao-Liang Zheng
}

Department of Chemistry and Chemical Biology, Harvard University, Cambridge, MA 02138, USA. *Correspondence e-mail: dkiper@fas.harvard.edu

The study of quadruple bonds between transition metals, in particular those of dimolybdenum, has revealed much about the two-electron bond. The solid-state structure of the quadruple-bonded dimolybdenum(II) complex tetrakis $[\mu-4-$ (trifluoromethyl)benzoato- $\kappa^{2} O: O^{\prime}$ ]bis[(tetrahydrofuran- $\kappa O$ )molybdenum(II)] 0.762-pentane 0.238-tetrahydrofuran solvate, $\left[\mathrm{Mo}_{2}\left(p-\mathrm{O}_{2} \mathrm{CC}_{6} \mathrm{H}_{4} \mathrm{CF}_{3}\right)_{4} \cdot 2 \mathrm{THF}\right] \cdot-$ $0.762 \mathrm{C}_{5} \mathrm{H}_{12} \cdot 0.238 \mathrm{C}_{4} \mathrm{H}_{8} \mathrm{O}$ or $\left[\mathrm{Mo}_{2}\left(\mathrm{C}_{8} \mathrm{H}_{4} \mathrm{~F}_{3} \mathrm{O}_{2}\right)_{4}\left(\mathrm{C}_{4} \mathrm{H}_{8} \mathrm{O}\right)_{2}\right] \cdot 0.762 \mathrm{C}_{5} \mathrm{H}_{12} \cdot 0.238 \mathrm{C}_{4} \mathrm{H}_{8} \mathrm{O}$ is reported. The complex crystallizes within a triclinic cell and low symmetry $(P \overline{1})$ results from the intercalated pentane/THF solvent molecules. The paddlewheel structure at $100 \mathrm{~K}$ has inversion symmetry and comprises four bridging carboxylate ligands encases the $\mathrm{Mo}_{2}$ (II,II) core that is characterized by two axially coordinated THF molecules and an Mo-Mo distance of 2.1098 (7) ̊.

\section{Chemical context}

The $\sigma^{2} \pi^{4} \delta^{2}$ quadruple bond has contributed prominently to the elucidation of the single most distinguishing feature of the discipline of chemistry - the two-electron bond (Lewis, 1916). As originally defined with the inception of valence and molecular orbital bonding models (Heitler \& London, 1927; Pauling, 1928; Lennard-Jones, 1929; Mulliken, 1932; James \& Coolidge, 1933; Coulson \& Fischer, 1949), the two-electron bond forms from pairing two electrons in two orbitals. Remarkably, the four states that characterize the two-electron bond remained undefined experimentally for over 60 years owing to the dissociative nature of the $\sigma$ and $\pi$ antibonding orbitals. This experimental challenge was overcome with the two-electron $\delta$ bond of quadruple-bonded metal-metal complexes. Anchored by a $\sigma^{2} \pi^{4}$ framework and sterically locking ligands, the four states of the two-electron bond, ${ }^{1} \delta \delta$, ${ }^{3} \delta \delta^{*},{ }^{1} \delta \delta^{*}$ and ${ }^{1} \delta^{*} \delta^{*}$, were experimentally defined for dimolybdenum quadruple-bond complexes (Engebretson et al. 1994, 1999; Cotton \& Nocera, 2000). Within the group of dimolybdenum quadruple-bond complexes, the tetraacetates are exemplars. The initial structure of $\mathrm{Mo}_{2}\left(\mathrm{O}_{2} \mathrm{CCH}_{3}\right)_{4}$ by Lawton \& Mason (1965) established the existence of the quadruple bond in the now familiar paddlewheel arrangement of acetates. The dimolybdenum bond distance of $2.11 \AA$ in this structure was subsequently refined nearly a decade later to be 2.0934 (8) (Cotton et al., 1974). Intriguingly, many subsequent structures have shown that the inductive effect of the $R$ group on the carboxylic acid does not perturb the Mo-Mo bond distance, indicating the robustness of the two-electron bond within a quadruple-bond architecture. It has been postulated 
that the strength of the Mo-Mo quadruple bond may be perturbed, but only in cases where $R$ is a strong electronwithdrawing group and there is a good axial donor ligand (Cotton et al., 1978). To add further to an understanding of $\mathrm{Mo}_{2}$ (II,II) quadruple bond distances, we examined a dimolybdenum core ligated by trifluoromethylbenzoate with THF axial donor ligands. We now report the synthesis and X-ray crystal structure of tetrakis( $\mu$-4-trifluoromethylbenzoato$\left.\kappa^{2} O: O^{\prime}\right)$ dimol-

ybdenum(II) 0.762-pentane 0.238-tetrahydrofuran solvate $\left[\mathrm{Mo}_{2}\left(p-\mathrm{O}_{2} \mathrm{CC}_{6} \mathrm{H}_{4} \mathrm{CF}_{3}\right)_{4} \cdot 2 \mathrm{THF}\right] \cdot 0.762 \mathrm{C}_{5} \mathrm{H}_{12} \cdot 0.238 \mathrm{C}_{4} \mathrm{H}_{8} \mathrm{O}$. The presence of the $\mathrm{CF}_{3}$ electron-withdrawing group on the bridging benzoate ligands, together with the donor THF axial ligands, results in a slightly elongated metal-metal bond distance as compared to its benzoate congener, $\mathrm{Mo}_{2}\left(\mathrm{O}_{2} \mathrm{CC}_{6} \mathrm{H}_{5}\right)_{4}$.

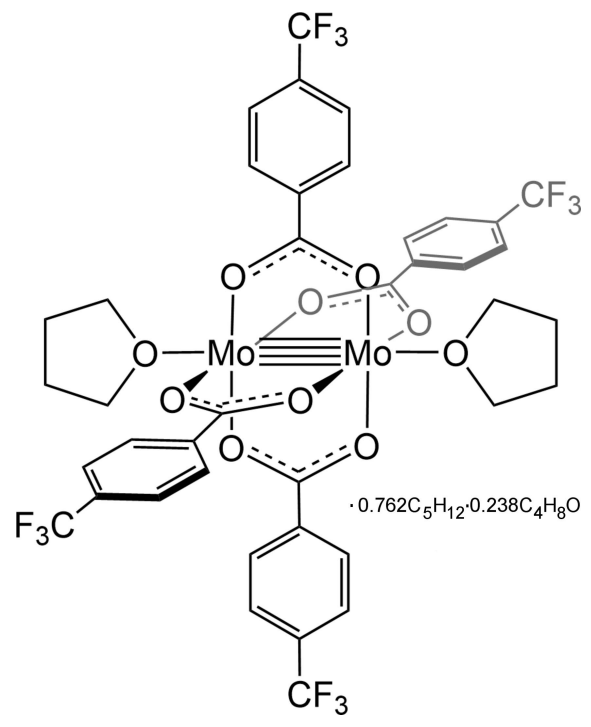

\section{Structural commentary}

The dimolybdenum complex, $\left[\mathrm{Mo}_{2}\left(p-\mathrm{O}_{2} \mathrm{CC}_{6} \mathrm{H}_{4} \mathrm{CF}_{3}\right)_{4} \cdot 2 \mathrm{THF}\right]$ (Fig. 1), was characterized by using single-crystal X-ray diffraction. Half of the molecule (Fig. 1) resides in the asymmetric unit, with the complete molecule generated by inversion about the quadruple-bond inversion center. The fluorine atoms of the trifluoromethyl groups are rotationally disordered and the highest occupancy positions are shown in Fig. 1. The crystallization solvents, THF and pentane, are disordered (0.238:0.762) (Fig. 2).

Selected bond metrics for $\mathrm{Mo}_{2}\left(p-\mathrm{O}_{2} \mathrm{CC}_{6} \mathrm{H}_{4} \mathrm{CF}_{3}\right)_{4} \cdot 2 \mathrm{THF}$ are listed in Table 1. Complete lists of the structural metrics for the compound are presented in the Supporting information. The Mo-Mo bond length is 2.1098 (7) $\AA$. Whereas the bond distance is within the typical range of dimolybdenum quadruple bond lengths of 2.06-2.17 (Cotton et al., 2002), it is slightly longer than what is observed for dimolybdenum cores bridged by carboxylates. As a comparison, the dimolybdenum bond distance in the $\mathrm{Mo}_{2}\left(\mathrm{O}_{2} \mathrm{CC}_{6} \mathrm{H}_{5}\right)_{4}$ congener, is 2.096 (1) $\AA$. Thus, with the addition of a $\mathrm{CF}_{3}$ group in the 4-position of benzoate, the Mo-Mo bond length increases by 0.014 (2) $\AA$.
Table 1

Selected geometric parameters $\left(\AA{ }^{\circ}\right)$.

\begin{tabular}{|c|c|c|c|}
\hline Mo1-O1 & $2.0996(17)$ & Mo1-Mo1 ${ }^{\mathrm{i}}$ & $2.1098(7)$ \\
\hline $\mathrm{Mo} 1-\mathrm{O} 4$ & 2.1030 (17) & $\mathrm{Mo} 1-\mathrm{O}^{\mathrm{i}}$ & 2.1204 (17) \\
\hline $\mathrm{Mo} 1-\mathrm{O} 2^{\mathrm{i}}$ & $2.1076(17)$ & Mo1-O1S & $2.5422(19)$ \\
\hline $\mathrm{O} 1-\mathrm{Mo1}-\mathrm{Mo}^{\mathrm{i}}$ & $93.20(5)$ & $\mathrm{O} 2^{\mathrm{i}}-\mathrm{Mo} 1-\mathrm{Mo}^{\mathrm{i}}$ & $90.10(5)$ \\
\hline $\mathrm{O} 4-\mathrm{Mo} 1-\mathrm{Mo}^{\mathrm{i}}$ & $92.37(5)$ & $\mathrm{Mo}^{\mathrm{i}}-\mathrm{Mo} 1-\mathrm{O}^{\mathrm{i}}$ & $90.84(5)$ \\
\hline
\end{tabular}

Symmetry code: (i) $-x,-y+1,-z+1$.

A similar trend is observed for the bond distances in the primary coordination sphere. The minimum $\mathrm{Mo}-\mathrm{O}$ bond distance decreases by 0.008 (5) $\AA$, and the maximum $\mathrm{Mo}-\mathrm{O}$ bond distance decreases by 0.011 (5) $\AA$ as compared to $\mathrm{Mo}_{2}\left(\mathrm{O}_{2} \mathrm{CC}_{6} \mathrm{H}_{5}\right)_{4}$. The most significant decrease in bond metrics is observed for the Mo-O1S axial ligand distance, which results in a decrease of 0.033 (4) $\AA$ for the axial coordinated oxygen atom of THF as compared to the axially coordinated oxygen in $\mathrm{Mo}_{2}\left(\mathrm{O}_{2} \mathrm{CC}_{6} \mathrm{H}_{5}\right)_{4}$. However, we note for this compound that the oxygen is provided from a carboxylate ligand of a neighboring molecule as opposed to an axially coordinated solvent molecule. Consequently, as proposed by Cotton (Cotton et al., 1978), the presence of ligands about the dimolybdenum center that are electron withdrawing and donating in the axial position is needed to perturb the overall bonding within a quadruple-bond framework. To this point, the metrics of $\left[\mathrm{Mo}_{2}\left(p-\mathrm{O}_{2} \mathrm{CC}_{6} \mathrm{H}_{4} \mathrm{CF}_{3}\right)_{4} \cdot \mathrm{THF}\right]$ are indistinguishable from those of $\mathrm{Mo}_{2}\left(\mathrm{O}_{2} \mathrm{CC}_{6} \mathrm{~F}_{5}\right)_{4}$. THF (Han, 2011). The electron-withdrawing nature of the fluoro-substituted benzoates is established by their $\mathrm{p} K_{\mathrm{a}} \mathrm{s}$ as compared to that of benzoate $\left(\mathrm{p} K_{\mathrm{a}}=1.75,3.77\right.$ and 4.20 for $\mathrm{C}_{6} \mathrm{~F}_{5} \mathrm{COOH}, p-\mathrm{CF}_{3}$ $\mathrm{C}_{6} \mathrm{H}_{4} \mathrm{COOH}$ and $\mathrm{C}_{6} \mathrm{H}_{5} \mathrm{COOH}$, respectively; Rumble, 2021; Boiadjiev \& Lightner, 1999). That an electron-withdrawing group alone is insufficient to perturb the dimolybdenum bond distance is indicated by a comparison of the structures for $\mathrm{Mo}_{2}\left(\mathrm{O}_{2} \mathrm{CCH}_{3}\right)_{4}$ and $\mathrm{Mo}_{2}\left(\mathrm{O}_{2} \mathrm{CCF}_{3}\right)_{4}$. The $d(\mathrm{Mo}-\mathrm{Mo})$ of 2.0934 (8) and $2.090(4) \AA$ for $\mathrm{Mo}_{2}\left(\mathrm{O}_{2} \mathrm{CCH}_{3}\right)_{4}$ and

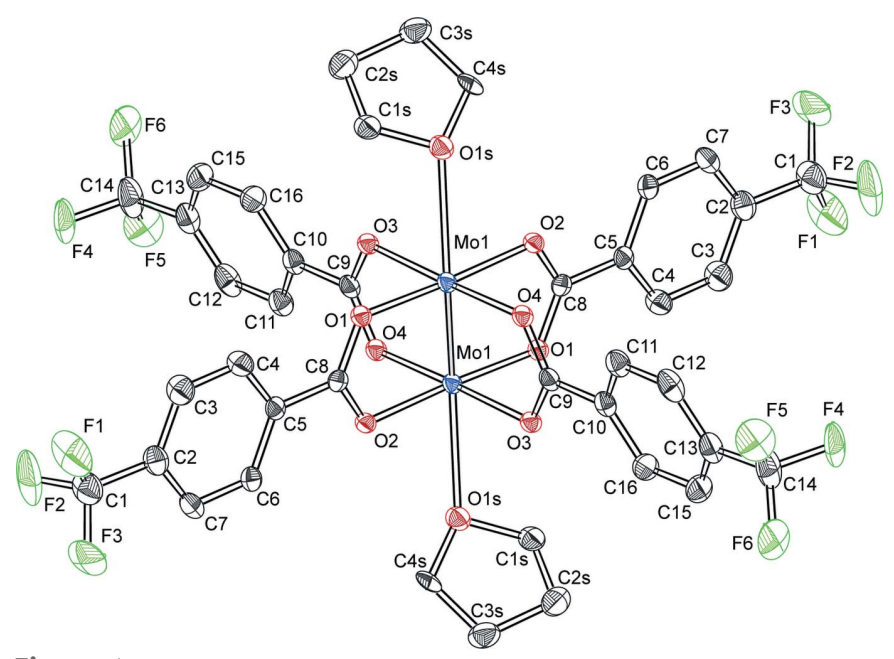

Figure 1

Ellipsoid plot of the dimolybdenum complex. The $\mathrm{CF}_{3}$ groups are rotationally disordered, therefore the highest occupancy positions are shown for each atom. Hydrogen atoms and unbound solvent are omitted for clarity. 
$\mathrm{Mo}_{2}\left(\mathrm{O}_{2} \mathrm{CCF}_{3}\right)_{4}$, respectively (Cotton \& Norman, 1971; Cotton et al., 1974), are indistinguishable despite a significant difference in electron-withdrawing properties $\left[\mathrm{p} K_{\mathrm{a}}\left(\mathrm{CH}_{3} \mathrm{COOH}\right)=\right.$ 4.76, $\mathrm{p} K_{\mathrm{a}}\left(\mathrm{CF}_{3} \mathrm{COOH}\right)=0.52$; Rumble, 2021]. Thus, a donor ligand is needed in addition to electron-withdrawing carboxylate equatorial ligands to observe a difference in the dimolybdenum quadruple bond.

\section{Supramolecular features}

The structure was solved in the triclinic space group $P \overline{1}$ with a half of an Mo-dimer per asymmetric unit and one full molecule per unit cell (Fig. 2). The low symmetry arises from the presence of disordered $\mathrm{THF} /$ pentane solvent molecules embedded within a solvent channel arising from the crystal packing. The disordered solvents are situated in the bodycenter of eight $\left[\mathrm{Mo}_{2}\left(p-\mathrm{O}_{2} \mathrm{CC}_{6} \mathrm{H}_{4} \mathrm{CF}_{3}\right)_{4} \cdot \mathrm{THF}\right]$ complexes with two THF molecules skewed towards the pentane; the next nearest neighbors are a series of four trifluoromethyl groups from distinct $\left[\mathrm{Mo}_{2}\left(p-\mathrm{O}_{2} \mathrm{CC}_{6} \mathrm{H}_{4} \mathrm{CF}_{3}\right)_{4} \cdot \mathrm{THF}\right]$ complexes. These four trifluoromethyl groups are oriented tangentially to the solvent channel (Fig. $2 b$ ) along the $b$-axis direction with a volume of $162 \AA^{3}$ for one void volume within the unit cell according to established methods for determining solventaccessible voids (van der Sluis \& Spek, 1990). The adjacent pairs of symmetry-related benzene rings $(\mathrm{C} 10-\mathrm{C} 16)$ in the $p-\mathrm{O}_{2} \mathrm{CC}_{6} \mathrm{H}_{4} \mathrm{CF}_{3}$ ligands interact through aromatic $\pi-\pi$ stacking interactions with a face-to-face distance of 3.7856 (9) $\AA$ (Fig. $2 b$ ) and form a one-dimensional chain. In addition, the trifluoromethyl group of a $p-\mathrm{O}_{2} \mathrm{CC}_{6} \mathrm{H}_{4} \mathrm{CF}_{3}$ ligand (for $\mathrm{C} 10-\mathrm{C} 16$ and $\mathrm{F} 4-\mathrm{F} 6$ ) is perpendicular to the aromatic plane of a neighboring $p-\mathrm{O}_{2} \mathrm{CC}_{6} \mathrm{H}_{4} \mathrm{CF}_{3}$ ligand $(\mathrm{C} 1-\mathrm{C} 7$ and $\mathrm{F} 1-$ $\mathrm{F} 3$ ) with weak $\mathrm{C}-\mathrm{F} \cdots \pi$ interactions (Kawahara et al., 2004) [the distances between the $\mathrm{F}$ atoms and the $\mathrm{C} 2-\mathrm{C} 8$ plane are 3.024 (2)-3.430 (1) $\AA$ ]. The coordinated THF molecules also have weak $\mathrm{C}-\mathrm{H} \cdots \mathrm{F}$ interactions (D'Oria \& Novoa, 2008) with the trifluoromethyl group of the $p-\mathrm{O}_{2} \mathrm{CC}_{6} \mathrm{H}_{4} \mathrm{CF}_{3}$ ligands [the $\mathrm{C}-\mathrm{H} \cdots \mathrm{F}$ distances are 2.568 (1)-3.045 (1) $\mathrm{A}$ ].

\section{Database survey}

In a search of the Cambridge Structural Database (WebCSD, accessed 17 December 2021; Groom et al., 2016), the CSD search fragment, $\mathrm{C}_{32} \mathrm{H}_{16} \mathrm{~F}_{12} \mathrm{Mo}_{2} \mathrm{O}_{8}$, for $\mathrm{Mo}_{2}\left(\mathrm{O}_{2} \mathrm{CC}_{6} \mathrm{H}_{4} \mathrm{CF}_{3}\right)_{4}$ yielded no hits in the database and the CSD search fragment, $\mathrm{C}_{40} \mathrm{H}_{32} \mathrm{~F}_{12} \mathrm{Mo}_{2} \mathrm{O}_{10}$, for $\left[\mathrm{Mo}_{2}\left(p-\mathrm{O}_{2} \mathrm{CC}_{6} \mathrm{H}_{4} \mathrm{CF}_{3}\right)_{4} \cdot \mathrm{THF}\right]$ also yielded no hits. The CSD reference code for $\mathrm{Mo}_{2}\left(\mathrm{O}_{2} \mathrm{CC}_{6} \mathrm{~F}_{5}\right)_{4} \cdot \mathrm{THF}$ (Han, 2011) is AYODOI, for $\mathrm{Mo}_{2}\left(\mathrm{O}_{2} \mathrm{CC}_{6} \mathrm{H}_{5}\right)_{4}$ (Cotton et al., 1978) is MOBZOA, for $\mathrm{Mo}_{2}\left(\mathrm{O}_{2} \mathrm{CCH}_{3}\right)_{4}$ (Cotton et al., 1974) is MOLACE01, and for $\mathrm{Mo}_{2}\left(\mathrm{O}_{2} \mathrm{CCF}_{3}\right)_{4}$ (Cotton \& Norman, 1971) is TFACMO.

\section{Purification and crystallization}

The overall synthetic scheme is shown in the reaction scheme. Molybdenum hexacarbonyl, 4-(trifluoromethyl) benzoic acid, THF, and 1,2-dichlorobenzene were purchased from Sigma-
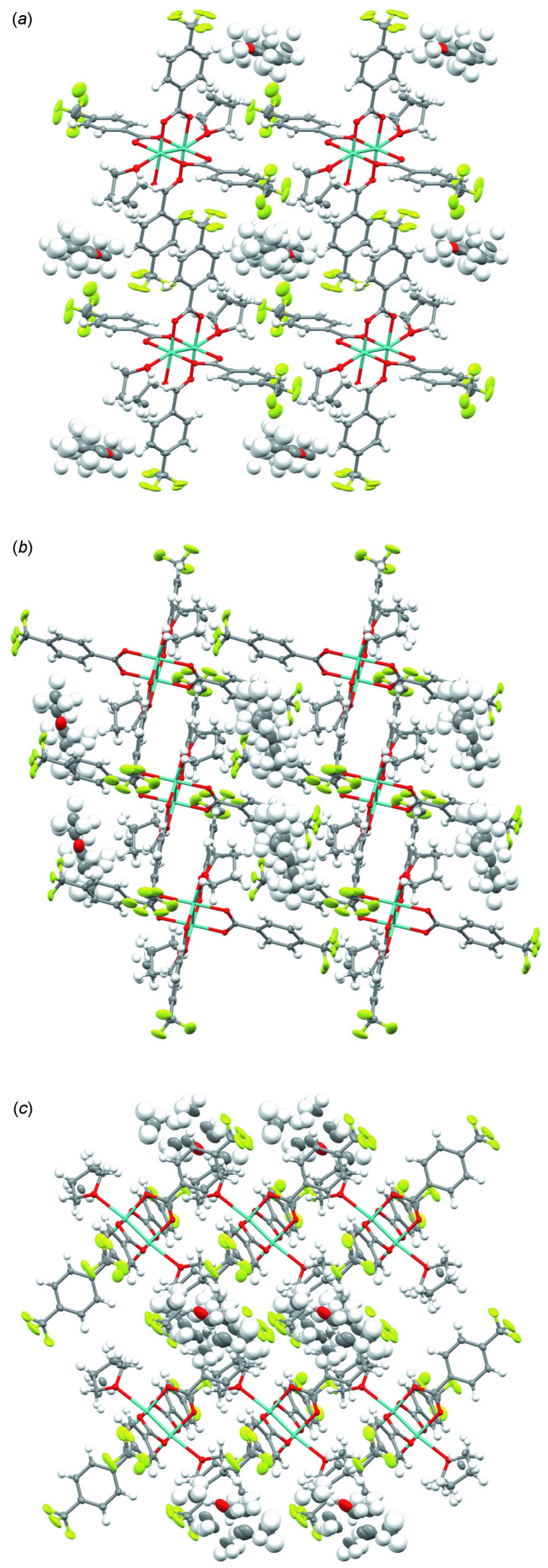

Figure 2

Crystal packing of the dimolybdenum complex shown along $(a)$ the $a$-axis, $(b)$ the $b$-axis and (c) the $c$-axis. The crystal has triclinic $(P \overline{1})$ symmetry. Pentane and THF solvent molecules are present in the structure. Color scheme: C (gray), O (red), H (white), F (green), Mo (teal). 
Aldrich. $\mathrm{Mo}(\mathrm{CO})_{6}$ and 4-(trifluoromethyl)benzoic acid were combined in a flask with THF and anhydrous 1,2-dichlorobenzene. The reaction was heated under reflux for $24 \mathrm{~h}$ at $413 \mathrm{~K}$ under nitrogen (Pence et al., 1999). The reaction mixture was cooled, the solution was filtered and the collected residue was washed with dichloromethane and hexanes.

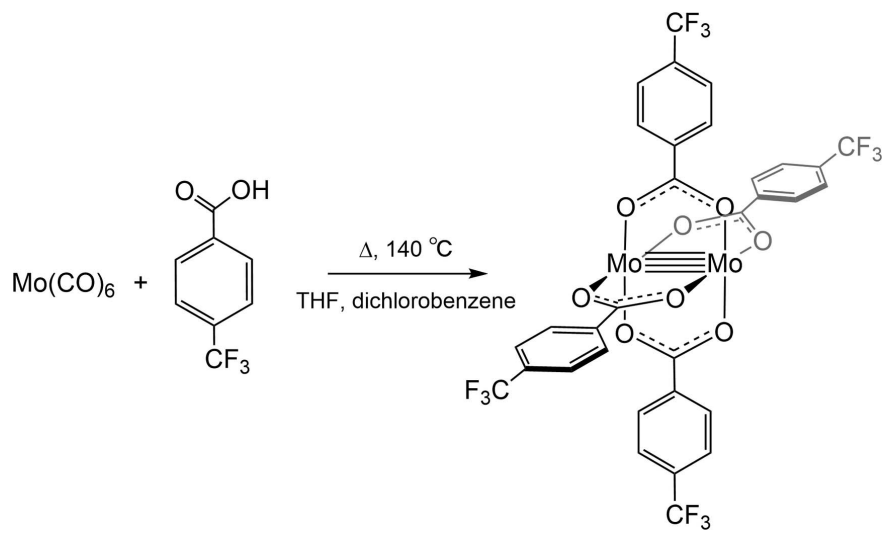

The crystallization was set up in a glove box. The crude product was dissolved in $\mathrm{THF}$ and recrystallized by vapor diffusion of pentane using a 6 by $50 \mathrm{~mm}$ borosilicate glass crystallization tube housed within a $20 \mathrm{~mL}$ glass vial. The assembly was allowed to stand at $238 \mathrm{~K}$ for 24 days. Orange rectangular crystals were observed and harvested for X-ray diffraction analysis.

\section{Refinement}

Crystal data, data collection and structure refinement details are included in Table 2. Hydrogen atoms on $\mathrm{C}$ atoms were placed at idealized positions and refined using a riding model. The isotropic displacement parameters of all hydrogen atoms were fixed to 1.2 times the atoms to which they are linked (1.5 times for methyl groups). Rotational and positional disorder for one trifluoromethyl substituent containing $\mathrm{C} 1$ and $\mathrm{C} 13$ was modeled. The overlapping solvent molecules (assigned as THF and pentane based on solvent crystallization conditions and apparent arrangement of electron-density peaks) were disordered adjacent to an inversion center (special position). The restraints on bond lengths and constraints of the atomic displacement parameters on each pair of disorder fragments (SADI/SAME and EADP instructions of SHELXL2014) as well as the restraints of the atomic displacement parameters (SIMU/RIGU instructions of SHELXL2014) were applied for the disorder refinement (Zheng et al., 2008). Crystallographic refinement details, including disorder modeling and the software employed, are given in the crystallographic information file (*.cif). To stabilize the refinement model, 713 restraints (SADI/SAME and RIGU/SIMU) were applied to accommodate the disordered trifluoromethyl group, the coordinated THF molecules, as well as the THF/pentane solvent molecules in the channel as detailed by Müller et al. (2006) to furnish a data+restraint-to-parameter ratio of 9.75. This ratio increases
Table 2

Experimental details.

\begin{tabular}{|c|c|}
\hline \multicolumn{2}{|l|}{ Crystal data } \\
\hline Chemical formula & $\begin{array}{c}{\left[\mathrm{Mo}_{2}\left(\mathrm{C}_{8} \mathrm{H}_{4} \mathrm{~F}_{3} \mathrm{O}_{2}\right)_{4}\left(\mathrm{C}_{4} \mathrm{H}_{8} \mathrm{O}\right)_{2}\right] \cdot-} \\
0.762 \mathrm{C}_{5} \mathrm{H}_{12} \cdot 0.238 \mathrm{C}_{4} \mathrm{H}_{8} \mathrm{O}\end{array}$ \\
\hline$M_{\mathrm{r}}$ & 1164.68 \\
\hline Crystal system, space group & Triclinic, $P \overline{1}$ \\
\hline Temperature (K) & 100 \\
\hline$a, b, c(\AA)$ & $7.7687(17), 12.099(5), 12.572(2)$ \\
\hline$\alpha, \beta, \gamma\left({ }^{\circ}\right)$ & $85.843(13), 81.208(8), 83.107(16)$ \\
\hline$V\left(\AA^{3}\right)$ & $1157.6(6)$ \\
\hline$Z$ & 1 \\
\hline Radiation type & Mo $K \alpha$ \\
\hline$\mu\left(\mathrm{mm}^{-1}\right)$ & 0.65 \\
\hline Crystal size $(\mathrm{mm})$ & $0.30 \times 0.13 \times 0.06$ \\
\hline \multicolumn{2}{|l|}{ Data collection } \\
\hline Diffractometer & $\begin{array}{l}\text { Bruker D8 goniometer with } \\
\text { Photon } 100 \text { CMOS detector }\end{array}$ \\
\hline Absorption correction & $\begin{array}{l}\text { Multi-scan (SADABS; Krause et } \\
\quad \text { al., 2015) }\end{array}$ \\
\hline$T_{\min }, T_{\max }$ & $0.701,0.745$ \\
\hline $\begin{array}{l}\text { No. of measured, independent and } \\
\text { observed }[I>2 \sigma(I)] \text { reflections }\end{array}$ & $39433,4094,3814$ \\
\hline$R_{\text {int }}$ & 0.033 \\
\hline$(\sin \theta / \lambda)_{\max }\left(\AA^{-1}\right)$ & 0.597 \\
\hline \multicolumn{2}{|l|}{ Refinement } \\
\hline$R\left[F^{2}>2 \sigma\left(F^{2}\right)\right], w R\left(F^{2}\right), S$ & $0.028,0.064,1.12$ \\
\hline No. of reflections & 4094 \\
\hline No. of parameters & 493 \\
\hline No. of restraints & 713 \\
\hline $\mathrm{H}$-atom treatment & $\mathrm{H}$-atom parameters constrained \\
\hline$\Delta \rho_{\max }, \Delta \rho_{\min }\left(\mathrm{e} \AA^{-3}\right)$ & $0.67,-0.39$ \\
\hline
\end{tabular}

Computer programs: APEX2 and SAINT (Bruker, 2015), SHELXT2014 (Sheldrick, 2015a), SHELXL2014 (Sheldrick, 2015b), SHELXTL (Sheldrick, 2008), and PLATON (Spek, 2020).

to 11.6 if the disordered $\mathrm{THF} /$ pentane solvent molecules in the channel are squeezed out of the structure.

\section{Acknowledgements}

We thank Theodore A. Betley and Daniel G. Nocera for helpful discussions and contributions to the preparation of the manuscript.

\section{Funding information}

Funding for this research was provided by: the Department of Chemistry and Chemical Biology, Harvard University.

\section{References}

Boiadjiev, S. E. \& Lightner, D. A. (1999). J. Phys. Org. Chem. 12, 751757.

Bruker (2015). APEX2 and SAINT. Bruker AXS Inc., Madison, Wisconsin, USA.

Cotton, F. A., Daniels, L. M., Hillard, E. A. \& Murillo, C. A. (2002). Inorg. Chem. 41, 2466-2470.

Cotton, F. A., Extine, M. \& Gage, L. D. (1978). Inorg. Chem. 17, 172176.

Cotton, F. A., Mester, Z. C. \& Webb, T. R. (1974). Acta Cryst. B30, 2768-2770.

Cotton, F. A. \& Nocera, D. G. (2000). Acc. Chem. Res. 33, 483-490.

Cotton, F. A. \& Norman, J. G. (1971). J. Coord. Chem. 1, 161-171.

Coulson, C. A. \& Fischer, I. (1949). Philos. Mag. 40, 386-393.

D’Oria, E. \& Novoa, J. J. (2008). CrystEngComm, 10, 423-436. 
Engebretson, D. S., Graj, E. M., Leroi, G. E. \& Nocera, D. G. (1999). J. Am. Chem. Soc. 121, 868-869.

Engebretson, D. S., Zaleski, J. M., Leroi, G. E. \& Nocera, D. G. (1994). Science, 265, 759-762.

Groom, C. R., Bruno, I. J., Lightfoot, M. P. \& Ward, S. C. (2016). Acta Cryst. B72, 171-179.

Han, L.-J. (2011). Acta Cryst. E67, m1289-m1290.

Heitler, W. \& London, F. (1927). Z. Phys. 44, 455-472.

James, H. M. \& Coolidge, A. S. (1933). J. Chem. Phys. 1, 825-835.

Kawahara, S., Tsuzuki, S. \& Uchimaru, T. (2004). J. Phys. Chem. A, 108, 6744-6749.

Krause, L., Herbst-Irmer, R., Sheldrick, G. M. \& Stalke, D. (2015). J. Appl. Cryst. 48, 3-10.

Lawton, D. \& Mason, R. (1965). J. Am. Chem. Soc. 87, 921-922.

Lennard-Jones, J. E. (1929). Trans. Faraday Soc. 25, 668-686.

Lewis, G. N. (1916). J. Am. Chem. Soc. 38, 762-785.
Müller, P., Herbst-Irmer, R., Spek, A., Schneider, T. \& Sawaya, M. (2006). Crystal Structure Refinement: a Crystallographer's Guide to SHELXL, p. 16. Oxford University Press.

Mulliken, R. S. (1932). Phys. Rev. 41, 751-758.

Pauling, L. (1928). Chem. Rev. 5, 173-213.

Pence, L. E., Weisgerber, A. M. \& Maounis, F. A. (1999). J. Chem. Educ. 76, 404-405.

Rumble, J. R. (2021). CRC Handbook of Chemistry and Physics, 102nd ed. Boca Raton: CRC Press.

Sheldrick, G. M. (2008). Acta Cryst. A64, 112-122.

Sheldrick, G. M. (2015a). Acta Cryst. C71, 3-8.

Sheldrick, G. M. (2015b). Acta Cryst. A71, 3-8.

Sluis, P. van der \& Spek, A. L. (1990). Acta Cryst. A46, 194-201.

Spek, A. L. (2020). Acta Cryst. E76, 1-11.

Zheng, S.-L., Vande Velde, C. M. L., Messerschmidt, M., Volkov, A., Gembicky, M. \& Coppens, P. (2008). Chem. Eur. J. 14, 706-713. 


\section{supporting information}

Acta Cryst. (2022). E78, 154-158 [https://doi.org/10.1107/S205698902200010X]

\section{Crystal structure of a trifluoromethyl benzoato quadruple-bonded dimolybdenum complex}

Elisabeth Aigeldinger, Lilliana Brandao, Troy Powell, Alaina C. Hartnett, Rui Sun, Dilek K. Dogutan and Shao-Liang Zheng

Computing details

Data collection: APEX2 (Bruker, 2015); cell refinement: SAINT (Bruker, 2015); data reduction: SAINT (Bruker, 2015); program(s) used to solve structure: SHELXT2014 (Sheldrick, 2015a); program(s) used to refine structure: SHELXL2014 (Sheldrick, 2015b); molecular graphics: SHELXTL (Sheldrick, 2008); software used to prepare material for publication: SHELXTL (Sheldrick, 2008), and PLATON (Spek, 2020).

Tetrakis[ $\mu$-4-(trifluoromethyl)benzoato- $\left.\kappa^{2} O: O^{\prime}\right)$ bis[(tetrahydrofuran- $\kappa O$ ) molybdenum(II)] 0.762-pentane 0.238 tetrahydrofuran solvate

Crystal data

$\left[\mathrm{Mo}_{2}\left(\mathrm{C}_{8} \mathrm{H}_{4} \mathrm{~F}_{3} \mathrm{O}_{2}\right)_{4}\left(\mathrm{C}_{4} \mathrm{H}_{8} \mathrm{O}\right)_{2}\right] \cdot 0.762 \mathrm{C}_{5} \mathrm{H}_{12} \cdot 0.238 \mathrm{C}_{4} \mathrm{H}_{8} \mathrm{O} \quad Z=1$

$M_{r}=1164.68$

Triclinic, $P \overline{1}$

$a=7.7687$ (17) $\AA$

$b=12.099(5) \AA$

$c=12.572(2) \AA$

$\alpha=85.843(13)^{\circ}$

$\beta=81.208(8)^{\circ}$

$\gamma=83.107(16)^{\circ}$

$F(000)=586$

$D_{\mathrm{x}}=1.671 \mathrm{Mg} \mathrm{m}^{-3}$

Mo $K \alpha$ radiation, $\lambda=0.71073 \AA$

Cell parameters from 9835 reflections

$\theta=2.5-27.2^{\circ}$

$\mu=0.65 \mathrm{~mm}^{-1}$

$T=100 \mathrm{~K}$

$V=1157.6(6) \AA^{3}$

Block, orange

$0.30 \times 0.13 \times 0.06 \mathrm{~mm}$

Data collection

Bruker D8 goniometer with Photon 100 CMOS detector diffractometer

Radiation source: $\mathrm{I} \mu \mathrm{S}$ microfocus tube $\omega$ and phi scans Absorption correction: multi-scan

(SADABS; Krause et al., 2015)

$T_{\min }=0.701, T_{\max }=0.745$

39433 measured reflections 4094 independent reflections 3814 reflections with $I>2 \sigma(I)$

$R_{\text {int }}=0.033$

$\theta_{\max }=25.1^{\circ}, \theta_{\min }=2.7^{\circ}$

$h=-9 \rightarrow 9$

$k=-14 \rightarrow 14$

$l=-14 \rightarrow 14$

Refinement

Refinement on $F^{2}$

Least-squares matrix: full

$R\left[F^{2}>2 \sigma\left(F^{2}\right)\right]=0.028$

$w R\left(F^{2}\right)=0.064$

$S=1.12$

4094 reflections

493 parameters

713 restraints

Primary atom site location: dual

Hydrogen site location: inferred from neighbouring sites

$\mathrm{H}$-atom parameters constrained 
$w=1 /\left[\sigma^{2}\left(F_{\mathrm{o}}^{2}\right)+(0.0191 P)^{2}+2.0296 P\right]$

where $P=\left(F_{\mathrm{o}}^{2}+2 F_{\mathrm{c}}^{2}\right) / 3$

$(\Delta / \sigma)_{\max }<0.001$

$$
\Delta \rho_{\max }=0.67 \mathrm{e} \AA^{-3}
$$$$
\Delta \rho_{\min }=-0.39 \text { e } \AA^{-3}
$$

\section{Special details}

Experimental. A single orange plate $(0.297 \mathrm{~mm} \times 0.132 \mathrm{~mm} \times 0.056 \mathrm{~mm})$ was chosen for single crystal X-ray diffraction using a Bruker three-circle platform goniometer equipped with an Photon100 CMOS detector. Data were collected as a series of $\varphi$ and/or $\omega$ scans. Data integration down to $0.84 \AA$ resolution was carried out using SAINT V8.37A with reflection spot size optimization. Absorption corrections were made with the program SADABS 2016/2 (Krause et al., 2015). Space group assignments were determined by examination of systematic absences, $E$-statistics, and successive refinement of the structures. The structure was solved by the Intrinsic Phasing methods and refined by least squares methods also using SHELXT-2014 and SHELXL-2014 with the OLEX 2 (Dolomanov et al., 2019) interface. The program PLATON (Spek, 2020) was employed to confirm the absence of higher symmetry space groups. All non-H atoms, including the disorder fragment, were located in difference Fourier maps, and then refined anisotropically. Outlier reflections were omitted from refinement when appropriate.

Geometry. All esds (except the esd in the dihedral angle between two 1.s. planes) are estimated using the full covariance matrix. The cell esds are taken into account individually in the estimation of esds in distances, angles and torsion angles; correlations between esds in cell parameters are only used when they are defined by crystal symmetry. An approximate (isotropic) treatment of cell esds is used for estimating esds involving l.s. planes.

Refinement. Refinement of $\mathrm{F}^{2}$ against ALL reflections. The weighted $\mathrm{R}$-factor $\mathrm{wR}$ and goodness of fit $\mathrm{S}$ are based on $\mathrm{F}^{2}$, conventional R-factors $R$ are based on $F$, with $F$ set to zero for negative $F^{2}$. The threshold expression of $F^{2}>2 \operatorname{sigma}\left(\mathrm{F}^{2}\right)$ is used only for calculating R-factors(gt) etc. and is not relevant to the choice of reflections for refinement. R-factors based on $\mathrm{F}^{2}$ are statistically about twice as large as those based on $\mathrm{F}$, and $\mathrm{R}$ - factors based on all data will be even larger. All non-H atoms, as well as the disordered atoms were located in difference-Fourier maps, and then refined anisotropically. The restraints on bond lengths and constraints of the atomic displacement parameters on each pair of disorder fragments (SADI/SAME and EADP instructions of SHELXL-2014) as well as the restraints of the atomic displacement parameters (SIMU/RIGU instructions of SHELXL- 2014), if necessary, have been applied for the disorder refinement.

Fractional atomic coordinates and isotropic or equivalent isotropic displacement parameters $\left(\AA^{2}\right)$

\begin{tabular}{llllll}
\hline & $x$ & $y$ & $z$ & $U_{\text {iso }} * / U_{\text {eq }}$ & Occ. $(<1)$ \\
\hline Mo1 & $-0.08669(3)$ & $0.43800(2)$ & $0.51162(2)$ & $0.01390(8)$ & $0.796(10)$ \\
C1 & $0.8554(11)$ & $0.0198(7)$ & $0.3139(9)$ & $0.0362(17)$ & $0.796(10)$ \\
F1 & $0.8182(10)$ & $-0.0767(5)$ & $0.2806(5)$ & $0.0615(15)$ & $0.796(10)$ \\
F2 & $0.9308(6)$ & $-0.0074(4)$ & $0.4035(4)$ & $0.0685(14)$ & $0.796(10)$ \\
F3 & $0.9798(5)$ & $0.0542(3)$ & $0.2420(4)$ & $0.0629(14)$ & $0.204(10)$ \\
C1A & $0.840(5)$ & $0.013(4)$ & $0.301(5)$ & $0.054(5)$ & $0.204(10)$ \\
F1A & $0.817(4)$ & $-0.083(2)$ & $0.327(2)$ & $0.066(5)$ & $0.204(10)$ \\
F2A & $0.9862(16)$ & $0.0311(12)$ & $0.3439(19)$ & $0.059(4)$ & $0.204(10)$ \\
F3A & $0.908(2)$ & $0.0366(15)$ & $0.1933(16)$ & $0.080(5)$ & $0.38(3)$ \\
C14 & $-0.273(2)$ & $0.6434(15)$ & $-0.1264(11)$ & $0.044(4)$ & $0.38(3)$ \\
F4 & $-0.421(2)$ & $0.7128(18)$ & $-0.1227(17)$ & $0.052(4)$ & $0.38(3)$ \\
F5 & $-0.303(3)$ & $0.5510(13)$ & $-0.1700(15)$ & $0.055(3)$ & $0.38(3)$ \\
F6 & $-0.1534(18)$ & $0.692(2)$ & $-0.1933(11)$ & $0.059(3)$ & $0.62(3)$ \\
C14A & $-0.2779(13)$ & $0.6523(8)$ & $-0.1247(6)$ & $0.034(2)$ & $0.62(3)$ \\
F4A & $-0.4522(11)$ & $0.6827(10)$ & $-0.1123(11)$ & $0.0398(19)$ & $0.62(3)$ \\
F5A & $-0.2538(17)$ & $0.5654(8)$ & $-0.1889(9)$ & $0.044(2)$ & $0.62(3)$ \\
F6A & $-0.1972(17)$ & $0.7326(11)$ & $-0.1815(8)$ & $0.053(2)$ & $0.0156(4)$ \\
O1 & $0.1106(2)$ & $0.31186(14)$ & $0.45777(13)$ & $0.0154(4)$ & \\
O2 & $0.2954(2)$ & $0.44209(14)$ & $0.43387(13)$ &
\end{tabular}




\begin{tabular}{|c|c|c|c|c|c|}
\hline $\mathrm{O} 3$ & $0.0284(2)$ & $0.60139(14)$ & $0.32975(13)$ & $0.0159(4)$ & \\
\hline $\mathrm{O} 4$ & $-0.1488(2)$ & $0.46706(14)$ & $0.35423(13)$ & $0.0160(4)$ & \\
\hline $\mathrm{C} 2$ & $0.6966(4)$ & $0.1020(2)$ & $0.3359(2)$ & $0.0258(6)$ & \\
\hline $\mathrm{C} 3$ & $0.5312(4)$ & $0.0680(2)$ & $0.3649(2)$ & $0.0287(6)$ & \\
\hline $\mathrm{H} 3$ & 0.5155 & -0.0086 & 0.3645 & $0.034^{*}$ & \\
\hline $\mathrm{C} 4$ & $0.3887(4)$ & $0.1452(2)$ & $0.3945(2)$ & $0.0238(6)$ & \\
\hline $\mathrm{H} 4$ & 0.2752 & 0.1218 & 0.4143 & $0.029^{*}$ & \\
\hline $\mathrm{C} 5$ & $0.4124(3)$ & $0.2572(2)$ & $0.39516(19)$ & $0.0169(5)$ & \\
\hline C6 & $0.5781(3)$ & $0.2909(2)$ & $0.3642(2)$ & $0.0189(5)$ & \\
\hline H6 & 0.5937 & 0.3677 & 0.3635 & $0.023 *$ & \\
\hline $\mathrm{C} 7$ & $0.7206(3)$ & $0.2141(2)$ & $0.3345(2)$ & $0.0231(6)$ & \\
\hline $\mathrm{H} 7$ & 0.8337 & 0.2376 & 0.3133 & $0.028^{*}$ & \\
\hline $\mathrm{C} 8$ & $0.2638(3)$ & $0.3418(2)$ & $0.43068(19)$ & $0.0159(5)$ & \\
\hline C9 & $-0.0782(3)$ & $0.5447(2)$ & $0.2967(2)$ & $0.0173(5)$ & \\
\hline $\mathrm{C} 10$ & $-0.1245(3)$ & $0.5704(2)$ & $0.1862(2)$ & $0.0190(5)$ & \\
\hline $\mathrm{C} 11$ & $-0.2181(3)$ & $0.4987(2)$ & $0.1418(2)$ & $0.0233(6)$ & \\
\hline H11 & -0.2507 & 0.4327 & 0.1815 & $0.028^{*}$ & \\
\hline $\mathrm{C} 12$ & $-0.2635(4)$ & $0.5235(3)$ & $0.0398(2)$ & $0.0284(7)$ & \\
\hline H12 & -0.3263 & 0.4743 & 0.0091 & $0.034 *$ & \\
\hline $\mathrm{C} 13$ & $-0.2173(4)$ & $0.6201(3)$ & $-0.0171(2)$ & $0.0282(7)$ & \\
\hline $\mathrm{C} 15$ & $-0.1237(4)$ & $0.6919(3)$ & $0.0260(2)$ & $0.0304(7)$ & \\
\hline H15 & -0.0920 & 0.7580 & -0.0138 & $0.036^{*}$ & \\
\hline $\mathrm{C} 16$ & $-0.0768(4)$ & $0.6667(2)$ & $0.1275(2)$ & $0.0250(6)$ & \\
\hline H16 & -0.0117 & 0.7153 & 0.1572 & $0.030^{*}$ & \\
\hline O1S & $-0.3188(2)$ & $0.31076(15)$ & $0.58783(14)$ & $0.0215(4)$ & $0.397(15)$ \\
\hline $\mathrm{C} 1 \mathrm{~S}$ & $-0.237(3)$ & $0.1999(13)$ & $0.6187(15)$ & 0.025 & $0.397(15)$ \\
\hline H1SA & -0.1085 & 0.1989 & 0.6102 & $0.030^{*}$ & $0.397(15)$ \\
\hline H1SB & -0.2658 & 0.1435 & 0.5728 & $0.030 *$ & $0.397(15)$ \\
\hline $\mathrm{C} 2 \mathrm{~S}$ & $-0.310(5)$ & $0.175(3)$ & $0.735(2)$ & $0.032(4)$ & $0.397(15)$ \\
\hline H2SA & -0.2329 & 0.1961 & 0.7844 & $0.038^{*}$ & $0.397(15)$ \\
\hline $\mathrm{H} 2 \mathrm{SB}$ & -0.3254 & 0.0946 & 0.7494 & $0.038^{*}$ & $0.397(15)$ \\
\hline $\mathrm{C} 3 \mathrm{~S}$ & $-0.4855(15)$ & $0.2465(9)$ & $0.7481(8)$ & $0.033(3)$ & $0.397(15)$ \\
\hline H3SA & -0.5817 & 0.2005 & 0.7457 & $0.039^{*}$ & $0.397(15)$ \\
\hline $\mathrm{H} 3 \mathrm{SB}$ & -0.5062 & 0.2823 & 0.8180 & $0.039 *$ & $0.397(15)$ \\
\hline $\mathrm{C} 4 \mathrm{~S}$ & $-0.4781(18)$ & $0.3292(12)$ & $0.6608(11)$ & $0.020(3)$ & $0.397(15)$ \\
\hline H4SA & -0.5790 & 0.3272 & 0.6217 & $0.024^{*}$ & $0.397(15)$ \\
\hline H4SB & -0.4868 & 0.4040 & 0.6896 & $0.024 *$ & $0.397(15)$ \\
\hline O1T & $-0.3188(2)$ & $0.31076(15)$ & $0.58783(14)$ & $0.0215(4)$ & $0.603(15)$ \\
\hline $\mathrm{C} 1 \mathrm{~T}$ & $-0.2628(19)$ & $0.1954(9)$ & $0.6098(9)$ & $0.025(2)$ & $0.603(15)$ \\
\hline H1TA & -0.1365 & 0.1778 & 0.5831 & $0.030^{*}$ & $0.603(15)$ \\
\hline H1TB & -0.3303 & 0.1473 & 0.5756 & $0.030^{*}$ & $0.603(15)$ \\
\hline $\mathrm{C} 2 \mathrm{~T}$ & $-0.298(3)$ & $0.1793(19)$ & $0.7318(12)$ & $0.029(3)$ & $0.603(15)$ \\
\hline H2TA & -0.1874 & 0.1726 & 0.7628 & $0.035^{*}$ & $0.603(15)$ \\
\hline H2TB & -0.3569 & 0.1113 & 0.7536 & $0.035^{*}$ & $0.603(15)$ \\
\hline $\mathrm{C} 3 \mathrm{~T}$ & $-0.4179(10)$ & $0.2836(6)$ & $0.7699(4)$ & $0.0307(17)$ & $0.603(15)$ \\
\hline H3TA & -0.5269 & 0.2626 & 0.8142 & $0.037 *$ & $0.603(15)$ \\
\hline Н3ТВ & -0.3577 & 0.3276 & 0.8133 & $0.037^{*}$ & $0.603(15)$ \\
\hline $\mathrm{C} 4 \mathrm{~T}$ & $-0.4562(12)$ & $0.3465(8)$ & $0.6735(7)$ & $0.021(2)$ & $0.603(15)$ \\
\hline
\end{tabular}




$\begin{array}{llllll}\text { H4TA } & -0.4587 & 0.4273 & 0.6826 & 0.025^{*} & 0.603(15) \\ \text { H4TB } & -0.5717 & 0.3322 & 0.6568 & 0.025^{*} & 0.603(15) \\ \text { C5S } & 0.2278(18) & 0.8776(12) & -0.0236(14) & 0.077(4) & 0.381(5) \\ \text { H5SA } & 0.2614 & 0.8639 & -0.1002 & 0.116^{*} & 0.381(5) \\ \text { H5SB } & 0.0998 & 0.8845 & -0.0058 & 0.116^{*} & 0.381(5) \\ \text { H5SC } & 0.2789 & 0.8154 & 0.0200 & 0.116^{*} & 0.381(5) \\ \text { C6S } & 0.2985(18) & 0.9909(13) & 0.0015(11) & 0.070(3) & 0.381(5) \\ \text { H6SA } & 0.2682 & 1.0516 & -0.0519 & 0.084^{*} & 0.381(5) \\ \text { H6SB } & 0.2462 & 1.0141 & 0.0744 & 0.084^{*} & 0.381(5) \\ \text { C7S } & 0.5068(17) & 0.9639(12) & -0.0061(15) & 0.075(4) & 0.381(5) \\ \text { H7SA } & 0.5375 & 0.8957 & 0.0386 & 0.090^{*} & 0.381(5) \\ \text { H7SB } & 0.5615 & 0.9530 & -0.0816 & 0.090^{*} & 0.381(5) \\ \text { C8S } & 0.571(2) & 1.0696(17) & 0.038(3) & 0.090(5) & 0.381(5) \\ \text { H8SA } & 0.5284 & 1.0750 & 0.1161 & 0.108^{*} & 0.381(5) \\ \text { H8SB } & 0.5270 & 1.1391 & -0.0007 & 0.108^{*} & 0.381(5) \\ \text { C9S } & 0.781(2) & 1.049(2) & 0.0156(16) & 0.108(6) & 0.381(5) \\ \text { H9SA } & 0.8179 & 1.0141 & -0.0534 & 0.162^{*} & 0.381(5) \\ \text { H9SB } & 0.8235 & 0.9998 & 0.0736 & 0.162^{*} & 0.381(5) \\ \text { H9SC } & 0.8292 & 1.1204 & 0.0128 & 0.162^{*} & 0.381(5) \\ \text { O2S } & 0.443(5) & 0.928(3) & -0.016(5) & 0.076(6) & 0.119(5) \\ \text { C10S } & 0.337(5) & 1.033(3) & -0.030(4) & 0.079(6) & 0.119(5) \\ \text { H10A } & 0.2310 & 1.0377 & 0.0246 & 0.095^{*} & 0.119(5) \\ \text { H10B } & 0.2999 & 1.0399 & -0.1026 & 0.095^{*} & 0.119(5) \\ \text { C11S } & 0.447(5) & 1.125(2) & -0.019(3) & 0.074(6) & 0.119(5) \\ \text { H11A } & 0.3840 & 1.1782 & 0.0338 & 0.089^{*} & 0.119(5) \\ \text { H11B } & 0.4788 & 1.1652 & -0.0887 & 0.089^{*} & 0.119(5) \\ \text { C12S } & 0.610(7) & 1.062(4) & 0.023(7) & 0.087(7) & 0.119(5) \\ \text { H12A } & 0.7167 & 1.0959 & -0.0106 & 0.105^{*} & 0.119(5) \\ \text { H12B } & 0.5991 & 1.0646 & 0.1020 & 0.105^{*} & 0.119(5) \\ \text { C13S } & 0.617(5) & 0.950(3) & -0.008(4) & 0.082(7) & 0.119(5) \\ \text { H13A } & 0.6922 & 0.9400 & -0.0786 & 0.099^{*} & 0.119(5) \\ \text { H13B } & 0.6662 & 0.8971 & 0.0462 & 0.099^{*} & 0.119(5)\end{array}$

Atomic displacement parameters $\left(\AA^{2}\right)$

\begin{tabular}{lllllll}
\hline & $U^{11}$ & $U^{22}$ & $U^{33}$ & $U^{12}$ & $U^{13}$ & $U^{23}$ \\
\hline Mo1 & $0.01239(11)$ & $0.01700(12)$ & $0.01261(12)$ & $-0.00162(8)$ & $-0.00291(8)$ & $-0.00050(8)$ \\
C1 & $0.030(3)$ & $0.027(3)$ & $0.051(3)$ & $0.002(2)$ & $-0.003(2)$ & $-0.009(2)$ \\
F1 & $0.044(2)$ & $0.036(2)$ & $0.104(4)$ & $0.0089(15)$ & $-0.002(3)$ & $-0.040(3)$ \\
F2 & $0.058(2)$ & $0.075(3)$ & $0.064(2)$ & $0.0443(19)$ & $-0.0200(19)$ & $-0.0107(19)$ \\
F3 & $0.035(2)$ & $0.0470(17)$ & $0.092(3)$ & $0.0125(14)$ & $0.0261(19)$ & $-0.0070(18)$ \\
C1A & $0.030(8)$ & $0.048(8)$ & $0.084(9)$ & $0.013(6)$ & $-0.003(6)$ & $-0.033(7)$ \\
F1A & $0.033(7)$ & $0.043(6)$ & $0.116(13)$ & $0.016(5)$ & $0.003(9)$ & $-0.029(8)$ \\
F2A & $0.028(6)$ & $0.049(7)$ & $0.101(11)$ & $0.012(4)$ & $-0.011(6)$ & $-0.029(7)$ \\
F3A & $0.042(8)$ & $0.092(9)$ & $0.095(8)$ & $0.030(6)$ & $0.010(6)$ & $-0.029(6)$ \\
C14 & $0.035(6)$ & $0.070(6)$ & $0.021(7)$ & $0.018(4)$ & $-0.010(4)$ & $-0.003(5)$ \\
F4 & $0.043(5)$ & $0.081(7)$ & $0.022(4)$ & $0.033(5)$ & $-0.009(4)$ & $0.006(6)$ \\
F5 & $0.058(7)$ & $0.091(5)$ & $0.015(5)$ & $0.000(4)$ & $-0.008(5)$ & $-0.009(4)$
\end{tabular}




\begin{tabular}{|c|c|c|c|c|c|c|}
\hline F6 & $0.054(5)$ & $0.099(8)$ & $0.017(3)$ & $0.008(5)$ & $-0.009(3)$ & $0.017(5)$ \\
\hline $\mathrm{C} 14 \mathrm{~A}$ & $0.033(4)$ & $0.050(4)$ & $0.017(4)$ & $0.008(3)$ & $-0.007(3)$ & $-0.004(3)$ \\
\hline F4A & $0.032(2)$ & $0.060(4)$ & $0.025(3)$ & $0.013(2)$ & $-0.013(2)$ & $0.000(3)$ \\
\hline F5A & $0.048(4)$ & $0.064(3)$ & $0.016(3)$ & $0.022(3)$ & $-0.011(3)$ & $-0.014(2)$ \\
\hline F6A & $0.061(4)$ & $0.075(5)$ & $0.026(3)$ & $-0.017(3)$ & -0.020 & $0.021(3)$ \\
\hline O1 & $0.0144(9)$ & $0.0176(9)$ & $0.0153(9)$ & $-0.0026(7)$ & $-0.0035(7)$ & $0.0000(7)$ \\
\hline $\mathrm{O} 2$ & $0.0133(8)$ & $0.0179(9)$ & $0.0154(9)$ & $-0.0026(7)$ & $-0.0026(7)$ & $0.0001(7)$ \\
\hline $\mathrm{O} 3$ & $0.0141(8)$ & $0.0195(9)$ & $0.0146(9)$ & $-0.0032(7)$ & $-0.0033(7)$ & $-0.0001(7)$ \\
\hline $\mathrm{O} 4$ & $0.0144(8)$ & $0.0195(9)$ & $0.0149(9)$ & $-0.0025(7)$ & $-0.0040(7)$ & $-0.0005(7)$ \\
\hline $\mathrm{C} 2$ & $0.0243(15)$ & $0.0243(15)$ & $0.0277(15)$ & $0.0034(12)$ & $-0.0031(12)$ & $-0.0055(12)$ \\
\hline $\mathrm{C} 3$ & $0.0283(15)$ & $0.0207(14)$ & $0.0370(17)$ & $-0.0003(12)$ & $-0.0040(13)$ & $-0.0064(12)$ \\
\hline $\mathrm{C} 4$ & $0.0199(14)$ & $0.0250(15)$ & $0.0271(15)$ & $-0.0037(11)$ & $-0.0032(11)$ & $-0.0030(11)$ \\
\hline $\mathrm{C} 5$ & $0.0167(13)$ & $0.0216(13)$ & $0.0125(12)$ & $-0.0004(10)$ & $-0.0047(10)$ & $0.0005(10)$ \\
\hline C6 & $0.0183(13)$ & $0.0203(13)$ & $0.0185(13)$ & $-0.0005(10)$ & $-0.0058(10)$ & $-0.0001(10)$ \\
\hline $\mathrm{C} 7$ & $0.0155(13)$ & $0.0295(15)$ & $0.0240(14)$ & $-0.0001(11)$ & $-0.0032(11)$ & $-0.0030(11)$ \\
\hline $\mathrm{C} 8$ & $0.0171(13)$ & $0.0208(14)$ & $0.0105(12)$ & $-0.0029(10)$ & $-0.0051(10)$ & $0.0016(10)$ \\
\hline C9 & $0.0132(12)$ & $0.0203(13)$ & $0.0176(13)$ & $0.0018(10)$ & $-0.0014(10)$ & $-0.0023(10)$ \\
\hline $\mathrm{C} 10$ & $0.0159(13)$ & $0.0262(14)$ & $0.0136(12)$ & $0.0035(10)$ & $-0.0017(10)$ & $-0.0035(10)$ \\
\hline $\mathrm{C} 11$ & $0.0199(13)$ & $0.0299(15)$ & $0.0198(14)$ & $0.0007(11)$ & $-0.0039(11)$ & $-0.0031(11)$ \\
\hline $\mathrm{C} 12$ & $0.0217(14)$ & $0.0448(18)$ & $0.0206(14)$ & $0.0020(13)$ & $-0.0093(11)$ & $-0.0112(13)$ \\
\hline $\mathrm{C} 13$ & $0.0221(14)$ & $0.0449(18)$ & $0.0149(13)$ & $0.0080(13)$ & $-0.0029(11)$ & $-0.0025(12)$ \\
\hline $\mathrm{C} 15$ & $0.0299(16)$ & $0.0399(18)$ & $0.0187(14)$ & $0.0002(13)$ & $-0.0025(12)$ & $0.0068(12)$ \\
\hline $\mathrm{C} 16$ & $0.0246(14)$ & $0.0310(15)$ & $0.0195(14)$ & $-0.0032(12)$ & $-0.0045(11)$ & $0.0010(11)$ \\
\hline O1S & $0.0183(9)$ & $0.0200(9)$ & $0.0255(10)$ & $-0.0013(7)$ & $-0.0011(7)$ & $-0.0022(7)$ \\
\hline $\mathrm{C} 1 \mathrm{~S}$ & $0.021(5)$ & $0.016(4)$ & $0.037(5)$ & $-0.004(4)$ & $-0.001(4)$ & $-0.003(3)$ \\
\hline $\mathrm{C} 2 \mathrm{~S}$ & $0.035(6)$ & $0.028(6)$ & $0.032(5)$ & $-0.003(5)$ & $-0.003(4)$ & $0.004(4)$ \\
\hline $\mathrm{C} 3 \mathrm{~S}$ & $0.034(4)$ & $0.028(4)$ & $0.031(4)$ & $-0.002(3)$ & $0.009(3)$ & $0.002(3)$ \\
\hline $\mathrm{C} 4 \mathrm{~S}$ & $0.010(4)$ & $0.028(5)$ & $0.024(4)$ & $-0.008(3)$ & $-0.006(3)$ & $-0.005(3)$ \\
\hline $\mathrm{O} 1 \mathrm{~T}$ & $0.0183(9)$ & $0.0200(9)$ & $0.0255(10)$ & $-0.0013(7)$ & $-0.0011(7)$ & $-0.0022(7)$ \\
\hline $\mathrm{C} 1 \mathrm{~T}$ & $0.020(4)$ & $0.021(3)$ & $0.033(3)$ & $-0.005(2)$ & $0.001(3)$ & $-0.002(2)$ \\
\hline $\mathrm{C} 2 \mathrm{~T}$ & $0.031(4)$ & $0.022(4)$ & $0.033(4)$ & $0.002(4)$ & -0.009 & $0.005(3)$ \\
\hline $\mathrm{C} 3 \mathrm{~T}$ & $0.026(3)$ & $0.037(3)$ & $0.025(2)$ & $0.005(3)$ & $0.003(2)$ & $0.002(2)$ \\
\hline C4T & $0.015(3)$ & $0.021(3)$ & $0.027(3)$ & -0.005 & 0.003 & $-0.003(2)$ \\
\hline $\mathrm{C} 5 \mathrm{~S}$ & $0.080(8)$ & $0.080(8)$ & $0.064(8)$ & $0.009(6)$ & $-0.005(6)$ & $0.015(7)$ \\
\hline C6S & $0.082(8)$ & $0.075(8)$ & $0.042(6)$ & $0.015(6)$ & $-0.005(6)$ & $0.027(5)$ \\
\hline $\mathrm{C} 7 \mathrm{~S}$ & $0.086(8)$ & $0.071(8)$ & $0.056(6)$ & $0.013(7)$ & $-0.002(6)$ & $0.025(6)$ \\
\hline $\mathrm{C} 8 \mathrm{~S}$ & $0.101(9)$ & $0.083(8)$ & $0.076(11)$ & $0.012(7)$ & $-0.011(7)$ & $0.013(7)$ \\
\hline C9S & $0.109(10)$ & $0.130(15)$ & $0.079(11)$ & $0.002(9)$ & $-0.019(8)$ & $0.015(12)$ \\
\hline $\mathrm{O} 2 \mathrm{~S}$ & $0.087(11)$ & $0.079(10)$ & $0.049(11)$ & $0.025(9)$ & $-0.011(10)$ & $0.020(9)$ \\
\hline $\mathrm{C} 10 \mathrm{~S}$ & $0.094(12)$ & $0.078(11)$ & $0.052(11)$ & $0.022(10)$ & $-0.005(11)$ & $0.026(10)$ \\
\hline $\mathrm{C} 11 \mathrm{~S}$ & $0.092(12)$ & $0.067(11)$ & $0.051(11)$ & $0.026(10)$ & $-0.012(10)$ & $0.031(10)$ \\
\hline $\mathrm{C} 12 \mathrm{~S}$ & $0.101(13)$ & $0.084(11)$ & $0.067(12)$ & $0.013(11)$ & $-0.008(11)$ & $0.019(11)$ \\
\hline $\mathrm{C} 13 \mathrm{~S}$ & $0.087(12)$ & $0.080(11)$ & $0.072(11)$ & $0.014(11)$ & $-0.020(11)$ & $0.025(10)$ \\
\hline
\end{tabular}

Geometric parameters $\left(A,{ }^{\circ}\right)$

\begin{tabular}{llll}
\hline Mo1-O1 & $2.0996(17)$ & C1S-C2S & $1.512(13)$ \\
Mo1-O4 & $2.1030(17)$ & C1S-H1SA & 0.9900
\end{tabular}




\begin{tabular}{|c|c|c|c|}
\hline $\mathrm{Mo} 1-\mathrm{O} 2^{\mathrm{i}}$ & $2.1076(17)$ & $\mathrm{C} 1 \mathrm{~S}-\mathrm{H} 1 \mathrm{SB}$ & 0.9900 \\
\hline Mo1-Mo1 ${ }^{\mathrm{i}}$ & $2.1098(7)$ & $\mathrm{C} 2 \mathrm{~S}-\mathrm{C} 3 \mathrm{~S}$ & $1.519(17)$ \\
\hline $\mathrm{Mo1}-\mathrm{O}^{\mathrm{i}}$ & $2.1204(17)$ & $\mathrm{C} 2 \mathrm{~S}-\mathrm{H} 2 \mathrm{SA}$ & 0.9900 \\
\hline Mo1-O1T & $2.5422(19)$ & $\mathrm{C} 2 \mathrm{~S}-\mathrm{H} 2 \mathrm{SB}$ & 0.9900 \\
\hline Mo1-O1S & $2.5422(19)$ & $\mathrm{C} 3 \mathrm{~S}-\mathrm{C} 4 \mathrm{~S}$ & $1.430(12)$ \\
\hline $\mathrm{C} 1-\mathrm{F} 3$ & $1.304(12)$ & $\mathrm{C} 3 \mathrm{~S}-\mathrm{H} 3 \mathrm{SA}$ & 0.9900 \\
\hline $\mathrm{C} 1-\mathrm{F} 1$ & $1.345(11)$ & $\mathrm{C} 3 \mathrm{~S}-\mathrm{H} 3 \mathrm{SB}$ & 0.9900 \\
\hline $\mathrm{C} 1-\mathrm{F} 2$ & $1.349(11)$ & $\mathrm{C} 4 \mathrm{~S}-\mathrm{H} 4 \mathrm{SA}$ & 0.9900 \\
\hline $\mathrm{C} 1-\mathrm{C} 2$ & $1.493(6)$ & $\mathrm{C} 4 \mathrm{~S}-\mathrm{H} 4 \mathrm{SB}$ & 0.9900 \\
\hline $\mathrm{C} 1 \mathrm{~A}-\mathrm{F} 1 \mathrm{~A}$ & $1.21(7)$ & $\mathrm{O} 1 \mathrm{~T}-\mathrm{C} 1 \mathrm{~T}$ & $1.431(9)$ \\
\hline $\mathrm{C} 1 \mathrm{~A}-\mathrm{F} 2 \mathrm{~A}$ & $1.38(5)$ & $\mathrm{O} 1 \mathrm{~T}-\mathrm{C} 4 \mathrm{~T}$ & $1.447(7)$ \\
\hline $\mathrm{C} 1 \mathrm{~A}-\mathrm{F} 3 \mathrm{~A}$ & $1.40(7)$ & $\mathrm{C} 1 \mathrm{~T}-\mathrm{C} 2 \mathrm{~T}$ & $1.518(11)$ \\
\hline $\mathrm{C} 1 \mathrm{~A}-\mathrm{C} 2$ & $1.494(18)$ & $\mathrm{C} 1 \mathrm{~T}-\mathrm{H} 1 \mathrm{TA}$ & 0.9900 \\
\hline $\mathrm{C} 14-\mathrm{F} 6$ & $1.314(14)$ & $\mathrm{C} 1 \mathrm{~T}-\mathrm{H} 1 \mathrm{~TB}$ & 0.9900 \\
\hline $\mathrm{C} 14-\mathrm{F} 4$ & $1.341(13)$ & $\mathrm{C} 2 \mathrm{~T}-\mathrm{C} 3 \mathrm{~T}$ & $1.535(13)$ \\
\hline $\mathrm{C} 14-\mathrm{F} 5$ & $1.341(14)$ & $\mathrm{C} 2 \mathrm{~T}-\mathrm{H} 2 \mathrm{TA}$ & 0.9900 \\
\hline $\mathrm{C} 14-\mathrm{C} 13$ & $1.500(13)$ & $\mathrm{C} 2 \mathrm{~T}-\mathrm{H} 2 \mathrm{~TB}$ & 0.9900 \\
\hline $\mathrm{C} 14 \mathrm{~A}-\mathrm{F} 6 \mathrm{~A}$ & $1.326(8)$ & $\mathrm{C} 3 \mathrm{~T}-\mathrm{C} 4 \mathrm{~T}$ & $1.434(8)$ \\
\hline $\mathrm{C} 14 \mathrm{~A}-\mathrm{F} 4 \mathrm{~A}$ & $1.348(9)$ & C3T-H3TA & 0.9900 \\
\hline $\mathrm{C} 14 \mathrm{~A}-\mathrm{F} 5 \mathrm{~A}$ & $1.349(8)$ & $\mathrm{C} 3 \mathrm{~T}-\mathrm{H} 3 \mathrm{~TB}$ & 0.9900 \\
\hline $\mathrm{C} 14 \mathrm{~A}-\mathrm{C} 13$ & $1.509(8)$ & $\mathrm{C} 4 \mathrm{~T}-\mathrm{H} 4 \mathrm{TA}$ & 0.9900 \\
\hline $\mathrm{O} 1-\mathrm{C} 8$ & 1.275 & $\mathrm{C} 4 \mathrm{~T}-\mathrm{H} 4 \mathrm{~TB}$ & 0.9900 \\
\hline $\mathrm{O} 2-\mathrm{C} 8$ & $1.271(3)$ & $\mathrm{C} 5 \mathrm{~S}-\mathrm{C} 6 \mathrm{~S}$ & $1.605(14)$ \\
\hline $\mathrm{O} 2-\mathrm{Mo}^{\mathrm{i}}$ & $2.1076(17)$ & $\mathrm{C} 5 \mathrm{~S}-\mathrm{H} 5 \mathrm{SA}$ & 0.9800 \\
\hline $\mathrm{O} 3-\mathrm{C} 9$ & $1.269(3)$ & C5S-H5SB & 0.9800 \\
\hline $\mathrm{O} 3-\mathrm{Mo}^{\mathrm{i}}$ & $2.1204(17)$ & $\mathrm{C} 5 \mathrm{~S}-\mathrm{H} 5 \mathrm{SC}$ & 0.9800 \\
\hline $\mathrm{O} 4-\mathrm{C} 9$ & $1.270(3)$ & $\mathrm{C} 6 \mathrm{~S}-\mathrm{C} 7 \mathrm{~S}$ & $1.602(14)$ \\
\hline $\mathrm{C} 2-\mathrm{C} 3$ & $1.384(4)$ & $\mathrm{C} 6 \mathrm{~S}-\mathrm{H} 6 \mathrm{SA}$ & 0.9900 \\
\hline $\mathrm{C} 2-\mathrm{C} 7$ & $1.389(4)$ & $\mathrm{C} 6 \mathrm{~S}-\mathrm{H} 6 \mathrm{SB}$ & 0.9900 \\
\hline $\mathrm{C} 3-\mathrm{C} 4$ & $1.382(4)$ & $\mathrm{C} 7 \mathrm{~S}-\mathrm{C} 8 \mathrm{~S}$ & $1.590(15)$ \\
\hline $\mathrm{C} 3-\mathrm{H} 3$ & 0.9500 & $\mathrm{C} 7 \mathrm{~S}-\mathrm{H} 7 \mathrm{SA}$ & 0.9900 \\
\hline $\mathrm{C} 4-\mathrm{C} 5$ & $1.390(4)$ & $\mathrm{C} 7 \mathrm{~S}-\mathrm{H} 7 \mathrm{SB}$ & 0.9900 \\
\hline $\mathrm{C} 4-\mathrm{H} 4$ & 0.9500 & $\mathrm{C} 8 \mathrm{~S}-\mathrm{C} 9 \mathrm{~S}$ & $1.607(15)$ \\
\hline $\mathrm{C} 5-\mathrm{C} 6$ & $1.387(4)$ & $\mathrm{C} 8 \mathrm{~S}-\mathrm{H} 8 \mathrm{SA}$ & 0.9900 \\
\hline $\mathrm{C} 5-\mathrm{C} 8$ & $1.485(3)$ & $\mathrm{C} 8 \mathrm{~S}-\mathrm{H} 8 \mathrm{SB}$ & 0.9900 \\
\hline $\mathrm{C} 6-\mathrm{C} 7$ & $1.381(4)$ & C9S-H9SA & 0.9800 \\
\hline $\mathrm{C} 6-\mathrm{H} 6$ & 0.9500 & C9S-H9SB & 0.9800 \\
\hline $\mathrm{C} 7-\mathrm{H} 7$ & 0.9500 & $\mathrm{C} 9 \mathrm{~S}-\mathrm{H} 9 \mathrm{SC}$ & 0.9800 \\
\hline $\mathrm{C} 9-\mathrm{C} 10$ & $1.490(3)$ & $\mathrm{O} 2 \mathrm{~S}-\mathrm{C} 13 \mathrm{~S}$ & $1.425(18)$ \\
\hline $\mathrm{C} 10-\mathrm{C} 16$ & $1.391(4)$ & $\mathrm{O} 2 \mathrm{~S}-\mathrm{C} 10 \mathrm{~S}$ & $1.442(18)$ \\
\hline $\mathrm{C} 10-\mathrm{C} 11$ & $1.392(4)$ & $\mathrm{C} 10 \mathrm{~S}-\mathrm{C} 11 \mathrm{~S}$ & $1.505(18)$ \\
\hline $\mathrm{C} 11-\mathrm{C} 12$ & $1.385(4)$ & $\mathrm{C} 10 \mathrm{~S}-\mathrm{H} 10 \mathrm{~A}$ & 0.9900 \\
\hline $\mathrm{C} 11-\mathrm{H} 11$ & 0.9500 & $\mathrm{C} 10 \mathrm{~S}-\mathrm{H} 10 \mathrm{~B}$ & 0.9900 \\
\hline $\mathrm{C} 12-\mathrm{C} 13$ & $1.382(4)$ & $\mathrm{C} 11 \mathrm{~S}-\mathrm{C} 12 \mathrm{~S}$ & $1.53(2)$ \\
\hline $\mathrm{C} 12-\mathrm{H} 12$ & 0.9500 & C11S-H11A & 0.9900 \\
\hline $\mathrm{C} 13-\mathrm{C} 15$ & $1.385(4)$ & $\mathrm{C} 11 \mathrm{~S}-\mathrm{H} 11 \mathrm{~B}$ & 0.9900 \\
\hline $\mathrm{C} 15-\mathrm{C} 16$ & $1.383(4)$ & $\mathrm{C} 12 \mathrm{~S}-\mathrm{C} 13 \mathrm{~S}$ & $1.436(18)$ \\
\hline $\mathrm{C} 15-\mathrm{H} 15$ & 0.9500 & $\mathrm{C} 12 \mathrm{~S}-\mathrm{H} 12 \mathrm{~A}$ & 0.9900 \\
\hline
\end{tabular}




\begin{tabular}{|c|c|c|c|}
\hline $\mathrm{C} 16-\mathrm{H} 16$ & 0.9500 & $\mathrm{C} 12 \mathrm{~S}-\mathrm{H} 12 \mathrm{~B}$ & 0.9900 \\
\hline $\mathrm{O} 1 \mathrm{~S}-\mathrm{C} 4 \mathrm{~S}$ & $1.428(11)$ & $\mathrm{C} 13 \mathrm{~S}-\mathrm{H} 13 \mathrm{~A}$ & 0.9900 \\
\hline $\mathrm{O} 1 \mathrm{~S}-\mathrm{C} 1 \mathrm{~S}$ & $1.465(12)$ & $\mathrm{C} 13 \mathrm{~S}-\mathrm{H} 13 \mathrm{~B}$ & 0.9900 \\
\hline $\mathrm{O} 1-\mathrm{Mo1}-\mathrm{O} 4$ & $89.95(7)$ & $\mathrm{H} 1 \mathrm{SA}-\mathrm{C} 1 \mathrm{~S}-\mathrm{H} 1 \mathrm{SB}$ & 108.6 \\
\hline $\mathrm{O} 1-\mathrm{Mo} 1-\mathrm{O} 2^{\mathrm{i}}$ & $176.69(6)$ & $\mathrm{C} 1 \mathrm{~S}-\mathrm{C} 2 \mathrm{~S}-\mathrm{C} 3 \mathrm{~S}$ & $102.6(12)$ \\
\hline $\mathrm{O} 4-\mathrm{Mo} 1-\mathrm{O} 2^{\mathrm{i}}$ & $89.57(7)$ & $\mathrm{C} 1 \mathrm{~S}-\mathrm{C} 2 \mathrm{~S}-\mathrm{H} 2 \mathrm{SA}$ & 111.3 \\
\hline $\mathrm{O} 1-\mathrm{Mo1}-\mathrm{Mol}^{\mathrm{i}}$ & $93.20(5)$ & $\mathrm{C} 3 \mathrm{~S}-\mathrm{C} 2 \mathrm{~S}-\mathrm{H} 2 \mathrm{SA}$ & 111.3 \\
\hline $\mathrm{O} 4-\mathrm{Mo} 1-\mathrm{Mo}^{\mathrm{i}}$ & $92.37(5)$ & $\mathrm{C} 1 \mathrm{~S}-\mathrm{C} 2 \mathrm{~S}-\mathrm{H} 2 \mathrm{SB}$ & 111.3 \\
\hline $\mathrm{O} 2^{\mathrm{i}}-\mathrm{Mo} 1-\mathrm{Mo}^{\mathrm{i}}$ & $90.10(5)$ & $\mathrm{C} 3 \mathrm{~S}-\mathrm{C} 2 \mathrm{~S}-\mathrm{H} 2 \mathrm{SB}$ & 111.3 \\
\hline $\mathrm{O} 1-\mathrm{Mo} 1-\mathrm{O} 3^{\mathrm{i}}$ & $88.41(7)$ & $\mathrm{H} 2 \mathrm{SA}-\mathrm{C} 2 \mathrm{~S}-\mathrm{H} 2 \mathrm{SB}$ & 109.2 \\
\hline $\mathrm{O} 4-\mathrm{Mo} 1-\mathrm{O} 3^{\mathrm{i}}$ & $176.47(7)$ & $\mathrm{C} 4 \mathrm{~S}-\mathrm{C} 3 \mathrm{~S}-\mathrm{C} 2 \mathrm{~S}$ & $106.9(10)$ \\
\hline $\mathrm{O} 2^{\mathrm{i}}-\mathrm{Mo} 1-\mathrm{O}^{\mathrm{i}}$ & $91.89(6)$ & $\mathrm{C} 4 \mathrm{~S}-\mathrm{C} 3 \mathrm{~S}-\mathrm{H} 3 \mathrm{SA}$ & 110.3 \\
\hline $\mathrm{Mo1}^{\mathrm{i}}-\mathrm{Mo1}-\mathrm{O}^{\mathrm{i}}$ & $90.84(5)$ & $\mathrm{C} 2 \mathrm{~S}-\mathrm{C} 3 \mathrm{~S}-\mathrm{H} 3 \mathrm{SA}$ & 110.3 \\
\hline $\mathrm{O} 1-\mathrm{Mo1}-\mathrm{O} 1 \mathrm{~T}$ & $96.83(6)$ & $\mathrm{C} 4 \mathrm{~S}-\mathrm{C} 3 \mathrm{~S}-\mathrm{H} 3 \mathrm{SB}$ & 110.3 \\
\hline $\mathrm{O} 4-\mathrm{Mo} 1-\mathrm{O} 1 \mathrm{~T}$ & $98.97(6)$ & $\mathrm{C} 2 \mathrm{~S}-\mathrm{C} 3 \mathrm{~S}-\mathrm{H} 3 \mathrm{SB}$ & 110.3 \\
\hline $\mathrm{O} 2^{\mathrm{i}}-\mathrm{Mo} 1-\mathrm{O} 1 \mathrm{~T}$ & $80.01(6)$ & $\mathrm{H} 3 \mathrm{SA}-\mathrm{C} 3 \mathrm{~S}-\mathrm{H} 3 \mathrm{SB}$ & 108.6 \\
\hline 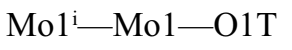 & $164.83(4)$ & $\mathrm{O} 1 \mathrm{~S}-\mathrm{C} 4 \mathrm{~S}-\mathrm{C} 3 \mathrm{~S}$ & $111.1(8)$ \\
\hline 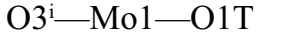 & $78.13(6)$ & $\mathrm{O} 1 \mathrm{~S}-\mathrm{C} 4 \mathrm{~S}-\mathrm{H} 4 \mathrm{SA}$ & 109.4 \\
\hline $\mathrm{O} 1-\mathrm{Mo1}-\mathrm{O} 1 \mathrm{~S}$ & $96.83(6)$ & $\mathrm{C} 3 \mathrm{~S}-\mathrm{C} 4 \mathrm{~S}-\mathrm{H} 4 \mathrm{SA}$ & 109.4 \\
\hline $\mathrm{O} 4-\mathrm{Mo} 1-\mathrm{O} 1 \mathrm{~S}$ & $98.97(6)$ & $\mathrm{O} 1 \mathrm{~S}-\mathrm{C} 4 \mathrm{~S}-\mathrm{H} 4 \mathrm{SB}$ & 109.4 \\
\hline $\mathrm{O} 2^{\mathrm{i}}-\mathrm{Mo} 1-\mathrm{O} 1 \mathrm{~S}$ & $80.01(6)$ & $\mathrm{C} 3 \mathrm{~S}-\mathrm{C} 4 \mathrm{~S}-\mathrm{H} 4 \mathrm{SB}$ & 109.4 \\
\hline Mo1 ${ }^{\mathrm{i}-\mathrm{Mo} 1-\mathrm{O} 1 \mathrm{~S}}$ & $164.83(4)$ & $\mathrm{H} 4 \mathrm{SA}-\mathrm{C} 4 \mathrm{~S}-\mathrm{H} 4 \mathrm{SB}$ & 108.0 \\
\hline 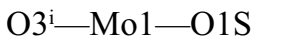 & $78.13(6)$ & $\mathrm{C} 1 \mathrm{~T}-\mathrm{O} 1 \mathrm{~T}-\mathrm{C} 4 \mathrm{~T}$ & $108.1(6)$ \\
\hline $\mathrm{F} 3-\mathrm{C} 1-\mathrm{F} 1$ & $106.4(6)$ & $\mathrm{C} 1 \mathrm{~T}-\mathrm{O} 1 \mathrm{~T}-\mathrm{Mo} 1$ & $118.0(6)$ \\
\hline $\mathrm{F} 3-\mathrm{C} 1-\mathrm{F} 2$ & $105.0(7)$ & $\mathrm{C} 4 \mathrm{~T}-\mathrm{O} 1 \mathrm{~T}-\mathrm{Mo} 1$ & $120.2(5)$ \\
\hline $\mathrm{F} 1-\mathrm{C} 1-\mathrm{F} 2$ & $106.0(8)$ & $\mathrm{O} 1 \mathrm{~T}-\mathrm{C} 1 \mathrm{~T}-\mathrm{C} 2 \mathrm{~T}$ & $104.5(9)$ \\
\hline $\mathrm{F} 3-\mathrm{C} 1-\mathrm{C} 2$ & $114.9(8)$ & $\mathrm{O} 1 \mathrm{~T}-\mathrm{C} 1 \mathrm{~T}-\mathrm{H} 1 \mathrm{TA}$ & 110.8 \\
\hline $\mathrm{F} 1-\mathrm{C} 1-\mathrm{C} 2$ & $112.5(7)$ & $\mathrm{C} 2 \mathrm{~T}-\mathrm{C} 1 \mathrm{~T}-\mathrm{H} 1 \mathrm{TA}$ & 110.8 \\
\hline $\mathrm{F} 2-\mathrm{C} 1-\mathrm{C} 2$ & $111.3(6)$ & $\mathrm{O} 1 \mathrm{~T}-\mathrm{C} 1 \mathrm{~T}-\mathrm{H} 1 \mathrm{~TB}$ & 110.8 \\
\hline $\mathrm{F} 1 \mathrm{~A}-\mathrm{C} 1 \mathrm{~A}-\mathrm{F} 2 \mathrm{~A}$ & $105(4)$ & $\mathrm{C} 2 \mathrm{~T}-\mathrm{C} 1 \mathrm{~T}-\mathrm{H} 1 \mathrm{~TB}$ & 110.8 \\
\hline $\mathrm{F} 1 \mathrm{~A}-\mathrm{C} 1 \mathrm{~A}-\mathrm{F} 3 \mathrm{~A}$ & $117(3)$ & $\mathrm{H} 1 \mathrm{TA}-\mathrm{C} 1 \mathrm{~T}-\mathrm{H} 1 \mathrm{~TB}$ & 108.9 \\
\hline $\mathrm{F} 2 \mathrm{~A}-\mathrm{C} 1 \mathrm{~A}-\mathrm{F} 3 \mathrm{~A}$ & $97(4)$ & $\mathrm{C} 1 \mathrm{~T}-\mathrm{C} 2 \mathrm{~T}-\mathrm{C} 3 \mathrm{~T}$ & $105.4(6)$ \\
\hline $\mathrm{F} 1 \mathrm{~A}-\mathrm{C} 1 \mathrm{~A}-\mathrm{C} 2$ & $118(5)$ & $\mathrm{C} 1 \mathrm{~T}-\mathrm{C} 2 \mathrm{~T}-\mathrm{H} 2 \mathrm{TA}$ & 110.7 \\
\hline $\mathrm{F} 2 \mathrm{~A}-\mathrm{C} 1 \mathrm{~A}-\mathrm{C} 2$ & $108(3)$ & $\mathrm{C} 3 \mathrm{~T}-\mathrm{C} 2 \mathrm{~T}-\mathrm{H} 2 \mathrm{TA}$ & 110.7 \\
\hline $\mathrm{F} 3 \mathrm{~A}-\mathrm{C} 1 \mathrm{~A}-\mathrm{C} 2$ & $109(4)$ & $\mathrm{C} 1 \mathrm{~T}-\mathrm{C} 2 \mathrm{~T}-\mathrm{H} 2 \mathrm{~TB}$ & 110.7 \\
\hline $\mathrm{F} 6-\mathrm{C} 14-\mathrm{F} 4$ & $106.6(13)$ & $\mathrm{C} 3 \mathrm{~T}-\mathrm{C} 2 \mathrm{~T}-\mathrm{H} 2 \mathrm{~TB}$ & 110.7 \\
\hline $\mathrm{F} 6-\mathrm{C} 14-\mathrm{F} 5$ & $108.3(13)$ & $\mathrm{H} 2 \mathrm{TA}-\mathrm{C} 2 \mathrm{~T}-\mathrm{H} 2 \mathrm{~TB}$ & 108.8 \\
\hline $\mathrm{F} 4-\mathrm{C} 14-\mathrm{F} 5$ & $106.3(15)$ & $\mathrm{C} 4 \mathrm{~T}-\mathrm{C} 3 \mathrm{~T}-\mathrm{C} 2 \mathrm{~T}$ & $105.6(7)$ \\
\hline $\mathrm{F} 6-\mathrm{C} 14-\mathrm{C} 13$ & $111.0(12)$ & $\mathrm{C} 4 \mathrm{~T}-\mathrm{C} 3 \mathrm{~T}-\mathrm{H} 3 \mathrm{TA}$ & 110.6 \\
\hline $\mathrm{F} 4-\mathrm{C} 14-\mathrm{C} 13$ & $111.7(14)$ & $\mathrm{C} 2 \mathrm{~T}-\mathrm{C} 3 \mathrm{~T}-\mathrm{H} 3 \mathrm{TA}$ & 110.6 \\
\hline $\mathrm{F} 5-\mathrm{C} 14-\mathrm{C} 13$ & $112.6(14)$ & $\mathrm{C} 4 \mathrm{~T}-\mathrm{C} 3 \mathrm{~T}-\mathrm{H} 3 \mathrm{~TB}$ & 110.6 \\
\hline $\mathrm{F} 6 \mathrm{~A}-\mathrm{C} 14 \mathrm{~A}-\mathrm{F} 4 \mathrm{~A}$ & $108.9(7)$ & $\mathrm{C} 2 \mathrm{~T}-\mathrm{C} 3 \mathrm{~T}-\mathrm{H} 3 \mathrm{~TB}$ & 110.6 \\
\hline $\mathrm{F} 6 \mathrm{~A}-\mathrm{C} 14 \mathrm{~A}-\mathrm{F} 5 \mathrm{~A}$ & $105.8(7)$ & $\mathrm{H} 3 \mathrm{TA}-\mathrm{C} 3 \mathrm{~T}-\mathrm{H} 3 \mathrm{~TB}$ & 108.7 \\
\hline $\mathrm{F} 4 \mathrm{~A}-\mathrm{C} 14 \mathrm{~A}-\mathrm{F} 5 \mathrm{~A}$ & $104.3(8)$ & $\mathrm{C} 3 \mathrm{~T}-\mathrm{C} 4 \mathrm{~T}-\mathrm{O} 1 \mathrm{~T}$ & $106.8(5)$ \\
\hline $\mathrm{F} 6 \mathrm{~A}-\mathrm{C} 14 \mathrm{~A}-\mathrm{C} 13$ & $114.5(7)$ & $\mathrm{C} 3 \mathrm{~T}-\mathrm{C} 4 \mathrm{~T}-\mathrm{H} 4 \mathrm{TA}$ & 110.4 \\
\hline $\mathrm{F} 4 \mathrm{~A}-\mathrm{C} 14 \mathrm{~A}-\mathrm{C} 13$ & $110.8(8)$ & $\mathrm{O} 1 \mathrm{~T}-\mathrm{C} 4 \mathrm{~T}-\mathrm{H} 4 \mathrm{TA}$ & 110.4 \\
\hline $\mathrm{F} 5 \mathrm{~A}-\mathrm{C} 14 \mathrm{~A}-\mathrm{C} 13$ & $112.0(8)$ & $\mathrm{C} 3 \mathrm{~T}-\mathrm{C} 4 \mathrm{~T}-\mathrm{H} 4 \mathrm{~TB}$ & 110.4 \\
\hline
\end{tabular}




\begin{tabular}{|c|c|}
\hline $\mathrm{C} 8-\mathrm{O} 1-\mathrm{Mo} 1$ & $115.86(15)$ \\
\hline $\mathrm{C} 8-\mathrm{O} 2-\mathrm{Mo}^{\mathrm{i}}$ & $118.49(15)$ \\
\hline $\mathrm{C} 9-\mathrm{O} 3-\mathrm{Mol}^{\mathrm{i}}$ & $117.28(15)$ \\
\hline $\mathrm{C} 9-\mathrm{O} 4-\mathrm{Mo} 1$ & $116.71(15)$ \\
\hline $\mathrm{C} 3-\mathrm{C} 2-\mathrm{C} 7$ & $120.3(3)$ \\
\hline $\mathrm{C} 3-\mathrm{C} 2-\mathrm{C} 1$ & $121.5(5)$ \\
\hline $\mathrm{C} 7-\mathrm{C} 2-\mathrm{C} 1$ & $118.0(5)$ \\
\hline $\mathrm{C} 3-\mathrm{C} 2-\mathrm{C} 1 \mathrm{~A}$ & $116(2)$ \\
\hline $\mathrm{C} 7-\mathrm{C} 2-\mathrm{C} 1 \mathrm{~A}$ & $124(3)$ \\
\hline $\mathrm{C} 4-\mathrm{C} 3-\mathrm{C} 2$ & $120.2(3)$ \\
\hline $\mathrm{C} 4-\mathrm{C} 3-\mathrm{H} 3$ & 119.9 \\
\hline $\mathrm{C} 2-\mathrm{C} 3-\mathrm{H} 3$ & 119.9 \\
\hline $\mathrm{C} 3-\mathrm{C} 4-\mathrm{C} 5$ & $119.7(3)$ \\
\hline $\mathrm{C} 3-\mathrm{C} 4-\mathrm{H} 4$ & 120.2 \\
\hline $\mathrm{C} 5-\mathrm{C} 4-\mathrm{H} 4$ & 120.2 \\
\hline $\mathrm{C} 6-\mathrm{C} 5-\mathrm{C} 4$ & $119.8(2)$ \\
\hline $\mathrm{C} 6-\mathrm{C} 5-\mathrm{C} 8$ & $119.0(2)$ \\
\hline $\mathrm{C} 4-\mathrm{C} 5-\mathrm{C} 8$ & $121.2(2)$ \\
\hline $\mathrm{C} 7-\mathrm{C} 6-\mathrm{C} 5$ & $120.7(2)$ \\
\hline $\mathrm{C} 7-\mathrm{C} 6-\mathrm{H} 6$ & 119.7 \\
\hline $\mathrm{C} 5-\mathrm{C} 6-\mathrm{H} 6$ & 119.7 \\
\hline $\mathrm{C} 6-\mathrm{C} 7-\mathrm{C} 2$ & $119.3(3)$ \\
\hline $\mathrm{C} 6-\mathrm{C} 7-\mathrm{H} 7$ & 120.4 \\
\hline $\mathrm{C} 2-\mathrm{C} 7-\mathrm{H} 7$ & 120.4 \\
\hline $\mathrm{O} 2-\mathrm{C} 8-\mathrm{O} 1$ & $122.3(2)$ \\
\hline $\mathrm{O} 2-\mathrm{C} 8-\mathrm{C} 5$ & $118.2(2)$ \\
\hline $\mathrm{O} 1-\mathrm{C} 8-\mathrm{C} 5$ & $119.5(2)$ \\
\hline $\mathrm{O} 3-\mathrm{C} 9-\mathrm{O} 4$ & $122.8(2)$ \\
\hline $\mathrm{O} 3-\mathrm{C} 9-\mathrm{C} 10$ & $119.1(2)$ \\
\hline $\mathrm{O} 4-\mathrm{C} 9-\mathrm{C} 10$ & $118.1(2)$ \\
\hline $\mathrm{C} 16-\mathrm{C} 10-\mathrm{C} 11$ & $119.7(2)$ \\
\hline $\mathrm{C} 16-\mathrm{C} 10-\mathrm{C} 9$ & $120.3(2)$ \\
\hline $\mathrm{C} 11-\mathrm{C} 10-\mathrm{C} 9$ & $120.0(2)$ \\
\hline $\mathrm{C} 12-\mathrm{C} 11-\mathrm{C} 10$ & $120.0(3)$ \\
\hline $\mathrm{C} 12-\mathrm{C} 11-\mathrm{H} 11$ & 120.0 \\
\hline $\mathrm{C} 10-\mathrm{C} 11-\mathrm{H} 11$ & 120.0 \\
\hline $\mathrm{C} 13-\mathrm{C} 12-\mathrm{C} 11$ & $119.8(3)$ \\
\hline $\mathrm{C} 13-\mathrm{C} 12-\mathrm{H} 12$ & 120.1 \\
\hline $\mathrm{C} 11-\mathrm{C} 12-\mathrm{H} 12$ & 120.1 \\
\hline $\mathrm{C} 12-\mathrm{C} 13-\mathrm{C} 15$ & $120.8(3)$ \\
\hline $\mathrm{C} 12-\mathrm{C} 13-\mathrm{C} 14$ & $117.3(7)$ \\
\hline $\mathrm{C} 15-\mathrm{C} 13-\mathrm{C} 14$ & $121.9(7)$ \\
\hline $\mathrm{C} 12-\mathrm{C} 13-\mathrm{C} 14 \mathrm{~A}$ & $120.1(5)$ \\
\hline $\mathrm{C} 15-\mathrm{C} 13-\mathrm{C} 14 \mathrm{~A}$ & $119.0(5)$ \\
\hline $\mathrm{C} 16-\mathrm{C} 15-\mathrm{C} 13$ & $119.5(3)$ \\
\hline $\mathrm{C} 16-\mathrm{C} 15-\mathrm{H} 15$ & 120.2 \\
\hline $\mathrm{C} 13-\mathrm{C} 15-\mathrm{H} 15$ & 120.2 \\
\hline $\mathrm{C} 15-\mathrm{C} 16-\mathrm{C} 10$ & $120.3(3)$ \\
\hline
\end{tabular}

\begin{tabular}{|c|c|}
\hline $\mathrm{O} 1 \mathrm{~T}-\mathrm{C} 4 \mathrm{~T}-\mathrm{H} 4 \mathrm{~TB}$ & 110.4 \\
\hline $\mathrm{H} 4 \mathrm{TA}-\mathrm{C} 4 \mathrm{~T}-\mathrm{H} 4 \mathrm{~TB}$ & 108.6 \\
\hline $\mathrm{C} 6 \mathrm{~S}-\mathrm{C} 5 \mathrm{~S}-\mathrm{H} 5 \mathrm{SA}$ & 109.5 \\
\hline $\mathrm{C} 6 \mathrm{~S}-\mathrm{C} 5 \mathrm{~S}-\mathrm{H} 5 \mathrm{SB}$ & 109.5 \\
\hline $\mathrm{H} 5 \mathrm{SA}-\mathrm{C} 5 \mathrm{~S}-\mathrm{H} 5 \mathrm{SB}$ & 109.5 \\
\hline $\mathrm{C} 6 \mathrm{~S}-\mathrm{C} 5 \mathrm{~S}-\mathrm{H} 5 \mathrm{SC}$ & 109.5 \\
\hline $\mathrm{H} 5 \mathrm{SA}-\mathrm{C} 5 \mathrm{~S}-\mathrm{H} 5 \mathrm{SC}$ & 109.5 \\
\hline $\mathrm{H} 5 \mathrm{SB}-\mathrm{C} 5 \mathrm{~S}-\mathrm{H} 5 \mathrm{SC}$ & 109.5 \\
\hline $\mathrm{C} 7 \mathrm{~S}-\mathrm{C} 6 \mathrm{~S}-\mathrm{C} 5 \mathrm{~S}$ & $106.0(10)$ \\
\hline $\mathrm{C} 7 \mathrm{~S}-\mathrm{C} 6 \mathrm{~S}-\mathrm{H} 6 \mathrm{SA}$ & 110.5 \\
\hline $\mathrm{C} 5 \mathrm{~S}-\mathrm{C} 6 \mathrm{~S}-\mathrm{H} 6 \mathrm{SA}$ & 110.5 \\
\hline $\mathrm{C} 7 \mathrm{~S}-\mathrm{C} 6 \mathrm{~S}-\mathrm{H} 6 \mathrm{SB}$ & 110.5 \\
\hline $\mathrm{C} 5 \mathrm{~S}-\mathrm{C} 6 \mathrm{~S}-\mathrm{H} 6 \mathrm{SB}$ & 110.5 \\
\hline $\mathrm{H} 6 \mathrm{SA}-\mathrm{C} 6 \mathrm{~S}-\mathrm{H} 6 \mathrm{SB}$ & 108.7 \\
\hline $\mathrm{C} 8 \mathrm{~S}-\mathrm{C} 7 \mathrm{~S}-\mathrm{C} 6 \mathrm{~S}$ & $105.2(12)$ \\
\hline $\mathrm{C} 8 \mathrm{~S}-\mathrm{C} 7 \mathrm{~S}-\mathrm{H} 7 \mathrm{SA}$ & 110.7 \\
\hline $\mathrm{C} 6 \mathrm{~S}-\mathrm{C} 7 \mathrm{~S}-\mathrm{H} 7 \mathrm{SA}$ & 110.7 \\
\hline $\mathrm{C} 8 \mathrm{~S}-\mathrm{C} 7 \mathrm{~S}-\mathrm{H} 7 \mathrm{SB}$ & 110.7 \\
\hline $\mathrm{C} 6 \mathrm{~S}-\mathrm{C} 7 \mathrm{~S}-\mathrm{H} 7 \mathrm{SB}$ & 110.7 \\
\hline $\mathrm{H} 7 \mathrm{SA}-\mathrm{C} 7 \mathrm{~S}-\mathrm{H} 7 \mathrm{SB}$ & 108.8 \\
\hline $\mathrm{C} 7 \mathrm{~S}-\mathrm{C} 8 \mathrm{~S}-\mathrm{C} 9 \mathrm{~S}$ & $104.7(14)$ \\
\hline $\mathrm{C} 7 \mathrm{~S}-\mathrm{C} 8 \mathrm{~S}-\mathrm{H} 8 \mathrm{SA}$ & 110.8 \\
\hline $\mathrm{C} 9 \mathrm{~S}-\mathrm{C} 8 \mathrm{~S}-\mathrm{H} 8 \mathrm{SA}$ & 110.8 \\
\hline $\mathrm{C} 7 \mathrm{~S}-\mathrm{C} 8 \mathrm{~S}-\mathrm{H} 8 \mathrm{SB}$ & 110.8 \\
\hline $\mathrm{C} 9 \mathrm{~S}-\mathrm{C} 8 \mathrm{~S}-\mathrm{H} 8 \mathrm{SB}$ & 110.8 \\
\hline $\mathrm{H} 8 \mathrm{SA}-\mathrm{C} 8 \mathrm{~S}-\mathrm{H} 8 \mathrm{SB}$ & 108.9 \\
\hline $\mathrm{C} 8 \mathrm{~S}-\mathrm{C} 9 \mathrm{~S}-\mathrm{H} 9 \mathrm{SA}$ & 109.5 \\
\hline $\mathrm{C} 8 \mathrm{~S}-\mathrm{C} 9 \mathrm{~S}-\mathrm{H} 9 \mathrm{SB}$ & 109.5 \\
\hline $\mathrm{H} 9 \mathrm{SA}-\mathrm{C} 9 \mathrm{~S}-\mathrm{H} 9 \mathrm{SB}$ & 109.5 \\
\hline $\mathrm{C} 8 \mathrm{~S}-\mathrm{C} 9 \mathrm{~S}-\mathrm{H} 9 \mathrm{SC}$ & 109.5 \\
\hline $\mathrm{H} 9 \mathrm{SA}-\mathrm{C} 9 \mathrm{~S}-\mathrm{H} 9 \mathrm{SC}$ & 109.5 \\
\hline $\mathrm{H} 9 \mathrm{SB}-\mathrm{C} 9 \mathrm{~S}-\mathrm{H} 9 \mathrm{SC}$ & 109.5 \\
\hline $\mathrm{C} 13 \mathrm{~S}-\mathrm{O} 2 \mathrm{~S}-\mathrm{C} 10 \mathrm{~S}$ & $108.7(18)$ \\
\hline $\mathrm{O} 2 \mathrm{~S}-\mathrm{C} 10 \mathrm{~S}-\mathrm{C} 11 \mathrm{~S}$ & $107.7(16)$ \\
\hline $\mathrm{O} 2 \mathrm{~S}-\mathrm{C} 10 \mathrm{~S}-\mathrm{H} 10 \mathrm{~A}$ & 110.2 \\
\hline $\mathrm{C} 11 \mathrm{~S}-\mathrm{C} 10 \mathrm{~S}-\mathrm{H} 10 \mathrm{~A}$ & 110.2 \\
\hline $\mathrm{O} 2 \mathrm{~S}-\mathrm{C} 10 \mathrm{~S}-\mathrm{H} 10 \mathrm{~B}$ & 110.2 \\
\hline $\mathrm{C} 11 \mathrm{~S}-\mathrm{C} 10 \mathrm{~S}-\mathrm{H} 10 \mathrm{~B}$ & 110.2 \\
\hline $\mathrm{H} 10 \mathrm{~A}-\mathrm{C} 10 \mathrm{~S}-\mathrm{H} 10 \mathrm{~B}$ & 108.5 \\
\hline $\mathrm{C} 10 \mathrm{~S}-\mathrm{C} 11 \mathrm{~S}-\mathrm{C} 12 \mathrm{~S}$ & $103.5(16)$ \\
\hline $\mathrm{C} 10 \mathrm{~S}-\mathrm{C} 11 \mathrm{~S}-\mathrm{H} 11 \mathrm{~A}$ & 111.1 \\
\hline $\mathrm{C} 12 \mathrm{~S}-\mathrm{C} 11 \mathrm{~S}-\mathrm{H} 11 \mathrm{~A}$ & 111.1 \\
\hline $\mathrm{C} 10 \mathrm{~S}-\mathrm{C} 11 \mathrm{~S}-\mathrm{H} 11 \mathrm{~B}$ & 111.1 \\
\hline $\mathrm{C} 12 \mathrm{~S}-\mathrm{C} 11 \mathrm{~S}-\mathrm{H} 11 \mathrm{~B}$ & 111.1 \\
\hline $\mathrm{H} 11 \mathrm{~A}-\mathrm{C} 11 \mathrm{~S}-\mathrm{H} 11 \mathrm{~B}$ & 109.0 \\
\hline $\mathrm{C} 13 \mathrm{~S}-\mathrm{C} 12 \mathrm{~S}-\mathrm{C} 11 \mathrm{~S}$ & $105.2(19)$ \\
\hline $\mathrm{C} 13 \mathrm{~S}-\mathrm{C} 12 \mathrm{~S}-\mathrm{H} 12 \mathrm{~A}$ & 110.7 \\
\hline $\mathrm{C} 11 \mathrm{~S}-\mathrm{C} 12 \mathrm{~S}-\mathrm{H} 12 \mathrm{~A}$ & 110.7 \\
\hline
\end{tabular}




\begin{tabular}{|c|c|c|c|}
\hline $\mathrm{C} 15-\mathrm{C} 16-\mathrm{H} 16$ & 119.9 & $\mathrm{C} 13 \mathrm{~S}-\mathrm{C} 12 \mathrm{~S}-\mathrm{H} 12 \mathrm{~B}$ & 110.7 \\
\hline $\mathrm{C} 10-\mathrm{C} 16-\mathrm{H} 16$ & 119.9 & $\mathrm{C} 11 \mathrm{~S}-\mathrm{C} 12 \mathrm{~S}-\mathrm{H} 12 \mathrm{~B}$ & 110.7 \\
\hline $\mathrm{C} 4 \mathrm{~S}-\mathrm{O} 1 \mathrm{~S}-\mathrm{C} 1 \mathrm{~S}$ & $106.0(9)$ & $\mathrm{H} 12 \mathrm{~A}-\mathrm{C} 12 \mathrm{~S}-\mathrm{H} 12 \mathrm{~B}$ & 108.8 \\
\hline $\mathrm{C} 4 \mathrm{~S}-\mathrm{O} 1 \mathrm{~S}-\mathrm{Mo} 1$ & $131.9(7)$ & $\mathrm{O} 2 \mathrm{~S}-\mathrm{C} 13 \mathrm{~S}-\mathrm{C} 12 \mathrm{~S}$ & $108(2)$ \\
\hline $\mathrm{C} 1 \mathrm{~S}-\mathrm{O} 1 \mathrm{~S}-\mathrm{Mo} 1$ & $110.8(10)$ & $\mathrm{O} 2 \mathrm{~S}-\mathrm{C} 13 \mathrm{~S}-\mathrm{H} 13 \mathrm{~A}$ & 110.1 \\
\hline $\mathrm{O} 1 \mathrm{~S}-\mathrm{C} 1 \mathrm{~S}-\mathrm{C} 2 \mathrm{~S}$ & $107.0(10)$ & $\mathrm{C} 12 \mathrm{~S}-\mathrm{C} 13 \mathrm{~S}-\mathrm{H} 13 \mathrm{~A}$ & 110.1 \\
\hline $\mathrm{O} 1 \mathrm{~S}-\mathrm{C} 1 \mathrm{~S}-\mathrm{H} 1 \mathrm{SA}$ & 110.3 & $\mathrm{O} 2 \mathrm{~S}-\mathrm{C} 13 \mathrm{~S}-\mathrm{H} 13 \mathrm{~B}$ & 110.1 \\
\hline $\mathrm{C} 2 \mathrm{~S}-\mathrm{C} 1 \mathrm{~S}-\mathrm{H} 1 \mathrm{SA}$ & 110.3 & $\mathrm{C} 12 \mathrm{~S}-\mathrm{C} 13 \mathrm{~S}-\mathrm{H} 13 \mathrm{~B}$ & 110.1 \\
\hline $\mathrm{O} 1 \mathrm{~S}-\mathrm{C} 1 \mathrm{~S}-\mathrm{H} 1 \mathrm{SB}$ & 110.3 & $\mathrm{H} 13 \mathrm{~A}-\mathrm{C} 13 \mathrm{~S}-\mathrm{H} 13 \mathrm{~B}$ & 108.4 \\
\hline $\mathrm{C} 2 \mathrm{~S}-\mathrm{C} 1 \mathrm{~S}-\mathrm{H} 1 \mathrm{SB}$ & 110.3 & & \\
\hline $\mathrm{F} 3-\mathrm{C} 1-\mathrm{C} 2-\mathrm{C} 3$ & $148.4(6)$ & $\mathrm{C} 11-\mathrm{C} 12-\mathrm{C} 13-\mathrm{C} 15$ & $0.9(4)$ \\
\hline $\mathrm{F} 1-\mathrm{C} 1-\mathrm{C} 2-\mathrm{C} 3$ & $26.5(11)$ & $\mathrm{C} 11-\mathrm{C} 12-\mathrm{C} 13-\mathrm{C} 14$ & $-179.0(8)$ \\
\hline $\mathrm{F} 2-\mathrm{C} 1-\mathrm{C} 2-\mathrm{C} 3$ & $-92.4(8)$ & $\mathrm{C} 11-\mathrm{C} 12-\mathrm{C} 13-\mathrm{C} 14 \mathrm{~A}$ & $-175.4(5)$ \\
\hline $\mathrm{F} 3-\mathrm{C} 1-\mathrm{C} 2-\mathrm{C} 7$ & $-36.4(9)$ & $\mathrm{F} 6-\mathrm{C} 14-\mathrm{C} 13-\mathrm{C} 12$ & $-144.3(13)$ \\
\hline $\mathrm{F} 1-\mathrm{C} 1-\mathrm{C} 2-\mathrm{C} 7$ & $-158.3(6)$ & $\mathrm{F} 4-\mathrm{C} 14-\mathrm{C} 13-\mathrm{C} 12$ & $96.8(18)$ \\
\hline $\mathrm{F} 2-\mathrm{C} 1-\mathrm{C} 2-\mathrm{C} 7$ & $82.8(9)$ & $\mathrm{F} 5-\mathrm{C} 14-\mathrm{C} 13-\mathrm{C} 12$ & $-22.7(17)$ \\
\hline $\mathrm{F} 1 \mathrm{~A}-\mathrm{C} 1 \mathrm{~A}-\mathrm{C} 2-\mathrm{C} 3$ & $-20(6)$ & $\mathrm{F} 6-\mathrm{C} 14-\mathrm{C} 13-\mathrm{C} 15$ & $35.7(17)$ \\
\hline $\mathrm{F} 2 \mathrm{~A}-\mathrm{C} 1 \mathrm{~A}-\mathrm{C} 2-\mathrm{C} 3$ & $-139(3)$ & $\mathrm{F} 4-\mathrm{C} 14-\mathrm{C} 13-\mathrm{C} 15$ & $-83.1(18)$ \\
\hline $\mathrm{F} 3 \mathrm{~A}-\mathrm{C} 1 \mathrm{~A}-\mathrm{C} 2-\mathrm{C} 3$ & $117(3)$ & $\mathrm{F} 5-\mathrm{C} 14-\mathrm{C} 13-\mathrm{C} 15$ & $157.3(12)$ \\
\hline $\mathrm{F} 1 \mathrm{~A}-\mathrm{C} 1 \mathrm{~A}-\mathrm{C} 2-\mathrm{C} 7$ & $164(4)$ & $\mathrm{F} 6 \mathrm{~A}-\mathrm{C} 14 \mathrm{~A}-\mathrm{C} 13-\mathrm{C} 12$ & $-166.7(8)$ \\
\hline $\mathrm{F} 2 \mathrm{~A}-\mathrm{C} 1 \mathrm{~A}-\mathrm{C} 2-\mathrm{C} 7$ & $44(6)$ & $\mathrm{F} 4 \mathrm{~A}-\mathrm{C} 14 \mathrm{~A}-\mathrm{C} 13-\mathrm{C} 12$ & $69.7(10)$ \\
\hline $\mathrm{F} 3 \mathrm{~A}-\mathrm{C} 1 \mathrm{~A}-\mathrm{C} 2-\mathrm{C} 7$ & $-60(4)$ & $\mathrm{F} 5 \mathrm{~A}-\mathrm{C} 14 \mathrm{~A}-\mathrm{C} 13-\mathrm{C} 12$ & $-46.3(11)$ \\
\hline $\mathrm{C} 7-\mathrm{C} 2-\mathrm{C} 3-\mathrm{C} 4$ & $-1.1(4)$ & $\mathrm{F} 6 \mathrm{~A}-\mathrm{C} 14 \mathrm{~A}-\mathrm{C} 13-\mathrm{C} 15$ & $16.9(10)$ \\
\hline $\mathrm{C} 1-\mathrm{C} 2-\mathrm{C} 3-\mathrm{C} 4$ & $174.0(5)$ & $\mathrm{F} 4 \mathrm{~A}-\mathrm{C} 14 \mathrm{~A}-\mathrm{C} 13-\mathrm{C} 15$ & $-106.7(9)$ \\
\hline $\mathrm{C} 1 \mathrm{~A}-\mathrm{C} 2-\mathrm{C} 3-\mathrm{C} 4$ & $-178(3)$ & $\mathrm{F} 5 \mathrm{~A}-\mathrm{C} 14 \mathrm{~A}-\mathrm{C} 13-\mathrm{C} 15$ & $137.3(8)$ \\
\hline $\mathrm{C} 2-\mathrm{C} 3-\mathrm{C} 4-\mathrm{C} 5$ & $-0.1(4)$ & $\mathrm{C} 12-\mathrm{C} 13-\mathrm{C} 15-\mathrm{C} 16$ & $-0.3(4)$ \\
\hline $\mathrm{C} 3-\mathrm{C} 4-\mathrm{C} 5-\mathrm{C} 6$ & $1.2(4)$ & $\mathrm{C} 14-\mathrm{C} 13-\mathrm{C} 15-\mathrm{C} 16$ & $179.6(8)$ \\
\hline $\mathrm{C} 3-\mathrm{C} 4-\mathrm{C} 5-\mathrm{C} 8$ & $-177.5(2)$ & $\mathrm{C} 14 \mathrm{~A}-\mathrm{C} 13-\mathrm{C} 15-\mathrm{C} 16$ & $176.0(5)$ \\
\hline $\mathrm{C} 4-\mathrm{C} 5-\mathrm{C} 6-\mathrm{C} 7$ & $-1.1(4)$ & $\mathrm{C} 13-\mathrm{C} 15-\mathrm{C} 16-\mathrm{C} 10$ & $-0.6(4)$ \\
\hline $\mathrm{C} 8-\mathrm{C} 5-\mathrm{C} 6-\mathrm{C} 7$ & $177.6(2)$ & $\mathrm{C} 11-\mathrm{C} 10-\mathrm{C} 16-\mathrm{C} 15$ & $0.8(4)$ \\
\hline $\mathrm{C} 5-\mathrm{C} 6-\mathrm{C} 7-\mathrm{C} 2$ & $-0.1(4)$ & $\mathrm{C} 9-\mathrm{C} 10-\mathrm{C} 16-\mathrm{C} 15$ & $-178.4(2)$ \\
\hline $\mathrm{C} 3-\mathrm{C} 2-\mathrm{C} 7-\mathrm{C} 6$ & $1.2(4)$ & $\mathrm{C} 4 \mathrm{~S}-\mathrm{O} 1 \mathrm{~S}-\mathrm{C} 1 \mathrm{~S}-\mathrm{C} 2 \mathrm{~S}$ & $-23(3)$ \\
\hline $\mathrm{C} 1-\mathrm{C} 2-\mathrm{C} 7-\mathrm{C} 6$ & $-174.1(5)$ & $\mathrm{Mo} 1-\mathrm{O} 1 \mathrm{~S}-\mathrm{C} 1 \mathrm{~S}-\mathrm{C} 2 \mathrm{~S}$ & $125(3)$ \\
\hline $\mathrm{C} 1 \mathrm{~A}-\mathrm{C} 2-\mathrm{C} 7-\mathrm{C} 6$ & $177(3)$ & $\mathrm{O} 1 \mathrm{~S}-\mathrm{C} 1 \mathrm{~S}-\mathrm{C} 2 \mathrm{~S}-\mathrm{C} 3 \mathrm{~S}$ & $25(4)$ \\
\hline $\mathrm{Mo}^{\mathrm{i}}-\mathrm{O} 2-\mathrm{C} 8-\mathrm{O} 1$ & $1.0(3)$ & $\mathrm{C} 1 \mathrm{~S}-\mathrm{C} 2 \mathrm{~S}-\mathrm{C} 3 \mathrm{~S}-\mathrm{C} 4 \mathrm{~S}$ & $-18(4)$ \\
\hline $\mathrm{Mo}^{\mathrm{i}}-\mathrm{O} 2-\mathrm{C} 8-\mathrm{C} 5$ & $-178.29(15)$ & $\mathrm{C} 1 \mathrm{~S}-\mathrm{O} 1 \mathrm{~S}-\mathrm{C} 4 \mathrm{~S}-\mathrm{C} 3 \mathrm{~S}$ & $11.2(18)$ \\
\hline $\mathrm{Mo} 1-\mathrm{O} 1-\mathrm{C} 8-\mathrm{O} 2$ & $-1.3(3)$ & $\mathrm{Mo} 1-\mathrm{O} 1 \mathrm{~S}-\mathrm{C} 4 \mathrm{~S}-\mathrm{C} 3 \mathrm{~S}$ & $-127.7(9)$ \\
\hline $\mathrm{Mo} 1-\mathrm{O} 1-\mathrm{C} 8-\mathrm{C} 5$ & $177.94(16)$ & $\mathrm{C} 2 \mathrm{~S}-\mathrm{C} 3 \mathrm{~S}-\mathrm{C} 4 \mathrm{~S}-\mathrm{O} 1 \mathrm{~S}$ & $5(3)$ \\
\hline $\mathrm{C} 6-\mathrm{C} 5-\mathrm{C} 8-\mathrm{O} 2$ & $-1.7(3)$ & $\mathrm{C} 4 \mathrm{~T}-\mathrm{O} 1 \mathrm{~T}-\mathrm{C} 1 \mathrm{~T}-\mathrm{C} 2 \mathrm{~T}$ & $-28.3(17)$ \\
\hline $\mathrm{C} 4-\mathrm{C} 5-\mathrm{C} 8-\mathrm{O} 2$ & $177.0(2)$ & $\mathrm{Mo} 1-\mathrm{O} 1 \mathrm{~T}-\mathrm{C} 1 \mathrm{~T}-\mathrm{C} 2 \mathrm{~T}$ & $112.4(15)$ \\
\hline $\mathrm{C} 6-\mathrm{C} 5-\mathrm{C} 8-\mathrm{O} 1$ & $179.0(2)$ & $\mathrm{O} 1 \mathrm{~T}-\mathrm{C} 1 \mathrm{~T}-\mathrm{C} 2 \mathrm{~T}-\mathrm{C} 3 \mathrm{~T}$ & $14(2)$ \\
\hline $\mathrm{C} 4-\mathrm{C} 5-\mathrm{C} 8-\mathrm{O} 1$ & $-2.3(4)$ & $\mathrm{C} 1 \mathrm{~T}-\mathrm{C} 2 \mathrm{~T}-\mathrm{C} 3 \mathrm{~T}-\mathrm{C} 4 \mathrm{~T}$ & $5(2)$ \\
\hline $\mathrm{Mo}^{\mathrm{i}}-\mathrm{O} 3-\mathrm{C} 9-\mathrm{O} 4$ & $-0.1(3)$ & $\mathrm{C} 2 \mathrm{~T}-\mathrm{C} 3 \mathrm{~T}-\mathrm{C} 4 \mathrm{~T}-\mathrm{O} 1 \mathrm{~T}$ & $-21.8(15)$ \\
\hline $\mathrm{Mo1}^{\mathrm{i}}-\mathrm{O} 3-\mathrm{C} 9-\mathrm{C} 10$ & $179.30(16)$ & $\mathrm{C} 1 \mathrm{~T}-\mathrm{O} 1 \mathrm{~T}-\mathrm{C} 4 \mathrm{~T}-\mathrm{C} 3 \mathrm{~T}$ & $32.4(11)$ \\
\hline $\mathrm{Mo} 1-\mathrm{O} 4-\mathrm{C} 9-\mathrm{O} 3$ & $1.7(3)$ & $\mathrm{Mo} 1-\mathrm{O} 1 \mathrm{~T}-\mathrm{C} 4 \mathrm{~T}-\mathrm{C} 3 \mathrm{~T}$ & $-107.3(6)$ \\
\hline $\mathrm{Mo} 1-\mathrm{O} 4-\mathrm{C} 9-\mathrm{C} 10$ & $-177.75(16)$ & $\mathrm{C} 5 \mathrm{~S}-\mathrm{C} 6 \mathrm{~S}-\mathrm{C} 7 \mathrm{~S}-\mathrm{C} 8 \mathrm{~S}$ & $-170.7(15)$ \\
\hline $\mathrm{O} 3-\mathrm{C} 9-\mathrm{C} 10-\mathrm{C} 16$ & $-11.2(4)$ & $\mathrm{C} 6 \mathrm{~S}-\mathrm{C} 7 \mathrm{~S}-\mathrm{C} 8 \mathrm{~S}-\mathrm{C} 9 \mathrm{~S}$ & $-172.8(17)$ \\
\hline
\end{tabular}


$\mathrm{O} 4-\mathrm{C} 9-\mathrm{C} 10-\mathrm{C} 16$

$\mathrm{O} 3-\mathrm{C} 9-\mathrm{C} 10-\mathrm{C} 11$

$\mathrm{O} 4-\mathrm{C} 9-\mathrm{C} 10-\mathrm{C} 11$

$\mathrm{C} 16-\mathrm{C} 10-\mathrm{C} 11-\mathrm{C} 12$

C9- $10-\mathrm{C} 11-\mathrm{C} 12$

$\mathrm{C} 10-\mathrm{C} 11-\mathrm{C} 12-\mathrm{C} 13$
$168.3(2)$

$169.7(2)$

-10.8 (3)

-0.2 (4)

$179.0(2)$

-0.7 (4)

Symmetry code: (i) $-x,-y+1,-z+1$.

$\begin{array}{ll}\mathrm{C} 13 \mathrm{~S}-\mathrm{O} 2 \mathrm{~S}-\mathrm{C} 10 \mathrm{~S}-\mathrm{C} 11 \mathrm{~S} & -6(6) \\ \mathrm{O} 2 \mathrm{~S}-\mathrm{C} 10 \mathrm{~S}-\mathrm{C} 11 \mathrm{~S}-\mathrm{C} 12 \mathrm{~S} & -10(5) \\ \mathrm{C} 10 \mathrm{~S}-\mathrm{C} 11 \mathrm{~S}-\mathrm{C} 12 \mathrm{~S}-\mathrm{C} 13 \mathrm{~S} & 22(6) \\ \mathrm{C} 10 \mathrm{~S}-\mathrm{O} 2 \mathrm{~S}-\mathrm{C} 13 \mathrm{~S}-\mathrm{C} 12 \mathrm{~S} & 21(7) \\ \mathrm{C} 11 \mathrm{~S}-\mathrm{C} 12 \mathrm{~S}-\mathrm{C} 13 \mathrm{~S}-\mathrm{O} 2 \mathrm{~S} & -27(7)\end{array}$

